\title{
Coordination of the Traditional and the Online Channels for a Short-life-cycle Product
}

\author{
Linghong Zhang* \\ School of Management Science and Engineering, \\ Shandong Normal University \\ Ji'nan, 250014, China \\ Email:zhanglinghong2005@126.com \\ Tel.: +86 15966304786, Fax: +86053186180510 \\ Jingguo Wang \\ Information Systems and Operations Management \\ College of Business, University of Texas at Arlington \\ Arlington TX, 76034 United States \\ Email:jwang@uta.edu
}

* Corresponding author 


\title{
Coordination of the Traditional and the Online Channels for a Short-life-cycle Product
}

\begin{abstract}
The study considers a retailer that sells a short-life-cycle product and uses the online channel as a supplement to its traditional channel. The inventory of the product is finite. The sales season is split into two periods: the premium period and the discount period. The study investigates how the retailer coordinates two channels with proper pricing strategies and channel mix. We introduce a new strategy to address channel conflict, in which two channels are introduced at different periods and the retailer employs different pricing mechanisms for two channels. We also explore the effects of return rate, service difference, and the retailer's risk attitude. Numerical examples are used to compare the solutions with other channel selection and pricing strategies, and further investigate the situations when some assumptions are not satisfied. The study suggests that the benefits of introducing the online channel are contingent on the potential demand of the online channel, the inventory level of the product, and the time of introducing the online channel. The retailer could adjust price difference between two channels to reduce channel conflict. Its risk attitude also influences the pricing strategies and sales revenue. Managerial implications of the study are discussed.
\end{abstract}

Key words: decision analysis, pricing, dual channels, channel conflict, risk attitude 


\section{Introduction}

Due to market competition and the speed of product development, developing a method of sellingproducts with a short-life cycle is a great challenge (Zhao \& Zheng, 2000). With the evolutionof the Internet, many firms (for example, apparel firms) take advantage of both the traditional, bricks-and-mortar channel and the online channel with a hope to reach more customers and boost sales. However, the online channel could exert competition pressure on existing bricks-and-mortar stores, resulting in channel conflict. The introduction of the online channel may lead to the closure of bricks-and-mortar retail stores (Yue \& Liu, 2006). Therefore, whether the retailer will benefit from the online channel, and how to address channel conflict, are questions calling for more studies (Yao \& Liu, 2005; Dan, Xu, \& Liu, 2012).

This study focuses on how a retailer that sells a perishable product can take advantage of both the traditional and the online channels. Specifically, we investigate the following research questions: (1) to mitigate channel conflict, when should the online channel be introduced and what are the pricing strategies that the retailer should use for these two channels? (2) How do return rate, service difference, and the retailer's risk attitude impact pricing, revenue, and channel conflict?

The modeling scenario of the study is drawn from the apparel industry. The apparel industry possesses seasonal and fashion characteristics (Johansen \& Riis, 1995). Many apparel retailers utilize both the traditional and the online channels to reach their customers and increase their sales (Chellappa, Sin, \& Siddarth, 2011). While the online channelenables retailers to reach more potential customersefficiently,introducingthe online channel does not always lead to better overall profitdue to channel conflict. Tomaximizeoverall profit and mitigate channel conflict, retailersoftenrely onstrategiessuch as differentiatingproduct pricesofthe two channels, or introducing valued-added services for the traditional channel.

In this study, the sales season of the short-life-cycle product is split into two periods: the premium period and the discount period. The price of the product decreases with its time on the market. This study introduces a new strategy to coordinate the two channels and mitigate channel conflict. In this strategy, only the traditional channel is utilized in the premium period, while both the traditional and the online channels are employed in the discount period. And the two channels adopt different pricing. We furtherdiscuss the scenario wherethe two channels are used in both periods in Subsection 6.2.2.We find that it does not always maximize revenue to introduce the short-life-cycle 
product in both the traditional and the online channels immediately.When the inventory level is small the retailer shall adopt the traditional channel alone. When the inventory is large, the retailer shallintroduce the online channel in the discount period. When the inventory is extremely large, the retailer shall introducethe two channels in both periods.

The study extends prior literature in three important aspects. First,priorstudieson the perishable products mainly explored dynamic pricing strategies butdid notinvestigate the impact of the online channel (Gallego \& Van Ryzin, 1994; Feng \& Gallego, 1995; Zhao \& Zheng, 2000;Aviv \& Pazgal, 2008). This study introduces a new selling strategy for perishablesin which the online channel is useda supplementto the traditional one. Second, most studies in dual channels (Chiang, Chhajed, \& Hess, 2003; Dumrongsiri et al. 2008 and Cai, Zhang, \& Zhang, 2009) focused on some general products other than perishables, andusedboth channels in the entire sale season.This studyintroduces a new strategy in which the retailer utilizes only the traditional channel in the premium period but both channels in the discount period to mitigate channel conflict, considering the characteristics of perishables. Third, we develop a dynamic pricing mechanism for the traditional channel to address channel conflict. Most studies on coordination of dual channels used static pricing mechanisms (e.g., Yao \& Liu, 2005; Dan, Xu, \& Liu, 2012).

The rest of the paper is organized as follows. Section 2 presents the related literature. Section 3 provides the modeling scenario and assumptions. Section 4 analyzes the basic and extended models with the consideration of return rate and service difference between two channels. Section 5 discusses the impact of demand uncertainty of the online channel and the retailer's risk attitude. Section 6 presents numerical examples and simulations to compare channel-selection and pricing strategies, and further explores the situations when some assumptions are violated. Section 7 discusses managerial implications, concludes the study and discusses future research.

\section{Related Literature}

In the apparel industry, a retailer often incorporates consumers' price sensitivity in its pricing strategyto maximize profit. Aviv \& Pazgal (2008) investigated optimal pricing strategies considering consumers' different reservation prices. Cachon \& Swinney (2009) studied the impact of dynamic pricing on consumers' strategic behavior. Li, Wei, \& Cai, (2012) explored how to sell new products and off-season products facing ahigh return rate in the apparel industry. In studying closed-loop 
supply chain models for a high-tech product which is featured with a short life-cycle and volatile demand,Chuang, Wang, \& Zhao (2014) presented the manufacturer's optimal production quantities and profits under the three alternative reverse channels for collecting the used product from consumers for remanufacturing. By setting different prices in the premium period and the discount period of the product, this study considers how the retailer can benefit from introducing the online channel as a supplement to the traditional channel. Broadly speaking, there are two streams of studies on channel selection related to this study.

The first stream examines the impact of introducing the online channel. Park \& Keh (2003) explored three scenarios: only using the online channel, only using its bricks-and-mortar channel, and using both channels. For each scenario, they analyzed the impact of cost structure and price sensitivity on equilibrium prices and revenue. Cattani, Perdikaki, \& Marucheck (2007) explored how to introduce the online channelfor grocery operations and suggested that the firm's channel selection depends on product price and quality. Dumrongsiri et al. (2008) examined the impact of channel marginal cost and demand uncertainty on product pricing and supply chain structure. It showed that the firm tends to add the online channel when wholesale price and demand uncertainty are low and marginal cost of the traditional channel is high. Considering the trade-off between risk pooling effect and transportation cost, Liu, Zhou, \& Zhang (2010) investigate how to assign online demands to the capacitated regional warehouses currently serving in-store demands in a multi-channel supply chain. Carrillo, Vakharia, \& Wang (2014) presented a dual-channel model for a retailer with online and traditional markets, and analyzed the impact of customer environmental sensitivity on retailer channel choice as well as stocking decisions for each channel. Cao, So, \& Yin (2016) added Online-to-Store channel (where customers order the product online and then pick up the product offline) and examined its impact on the demand allocations and profitability of a retailer who sells products through multiple distribution channels.

The second stream of literature primarily addresses how to solve channel conflict when a firm utilizes both the online and the traditional channels. Some literature relied on service difference between the two channels to address their conflict. Yan \& Pei (2009) showed that the online channel could encourage the retailer to improve service. Ofek, Katona, \& Sarvary (2011)incorporatedreturn rate to consumers' utility function, and analyzed the impact of return rate on channel prices. Chen \& Bell (2012) explored different return policies for two channels and showed that segmenting the 
market based on consumers’ sensitivity to return can be a profit-maximizing policy.

Some researchers also introduced product differentiation and/or price difference between two channels. Riggins (2004) developed a multi-channel model where a retailer sells a durable good to high- and low-type consumers in two different channels. Kurata, Yao, \& Liu (2007) studied the optimal prices when the online channel sells national brands, and the traditional channel sells both the national brands and the store brands. Yao \& Liu (2005) discussed the impact of price difference and customer service on the equilibrium prices. Marom \& Seidmann (2011) distinguished the channels by prices and availability of products.

Most studies differentiated the traditional channel and the online channel either by the price or by the product (including service). In this paper we not only introduce different pricing strategies for the two channels, but also engage the two channels in different periods: the online channel will only be used to sell the product in the discount period. Based on the characteristics of short-life-cycle products, we address channel conflict in the following ways: (1) product difference-the traditional channel sellsboth the new and off-season product, but the online channel only sells the off-season product (or the product in the discount period); (2) pricing difference-the traditional channel employs dynamic prices for the two periods, while the online channel employs static price (only in the discount period). Furthermore, we discuss the impact of service difference, return rate, and demand uncertainty.

\section{Problem Description}

Consider a retailer who sells a short-life-cycle product with the traditional channel. Because of the long production life cycle, the retailer has only one ordering opportunity for the sales season (Zhao \& Zheng, 2000). Therefore its inventory of the product is finite. We consider the retailer has $Q$ units of the product in its inventory. The sales season is split into two periods, namely period 1 (the premium period) and period 2 (the discount period) (Aviv \& Pazgal, 2008). In the first period, the retailer only utilizes the traditional channel (i.e., single channel mode) to sell the product. In the second period, the retailer introduces the online channel to sell the product together with the traditional channel. In other words, the product is sold via both channels in the discount period.We do not incorporatethe length of each period as a model parameter, but one may reasonably assume that the length of a period can be reflected by the corresponding potential market size ( $\left.a_{i j}\right)$ (Aviv \& Pazgal, 
2008).

\subsection{Demand Functions}

During the first period of the sales season, a 'premium price' $p_{11}$ isapplied in the traditional channel. During the second period of the season, a 'discount price' $p_{21}$ is offered in the traditional channel and $p_{22}$ in the online channel. The premium price $p_{11}$ is announced at the beginning of period 1 , and the discount pricesp $p_{21}$ and $p_{22}$ at the beginning of period 2.At the end of the sales season, the retailer clears the remaining inventory with a salvage value, and we assume that theunit loss of the product incurred by clearing is $v$. Because of the low cost of the online channel, we assume $p_{21} \geq p_{22}$ which is in accordance with the real world and prior studies (Yao \& Liu, 2005) . Fig.1 shows such a pricing policy.

add online channel

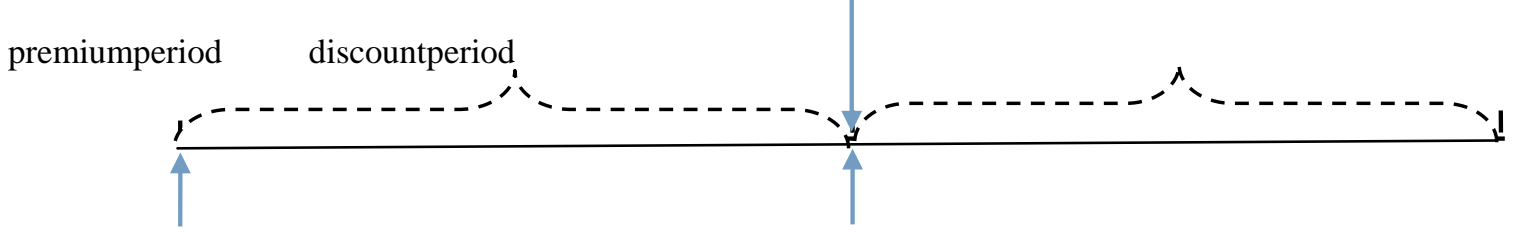

announce traditional channel price $p_{11}$ announce traditional and online channel prices $p_{21}, p_{22}$

Fig.1Dynamic pricing with dual channels

We assume that the demand in each period is price sensitive. Consumers can purchase the product from either channel. Similar toYao \& Liu (2005) and Dan, Xu, \& Liu (2012), we assume the demand function of the traditional channel in the premium period is $D_{11}=a_{11}-b_{1} p_{11}$. And in the discount period, the demand of the traditional channel is $D_{21}=a_{21}-b_{2} p_{21}+\theta\left(p_{22}-p_{21}\right)$. The demand of the online channel in the discount period is $D_{22}=a_{22}-b_{2} p_{22}+\theta\left(p_{21}-p_{22}\right) . a_{i j}$ in the above demand equationsrepresents the potential demand in period $i$ channel $j(i=1$ or $2, j=1$ or 2$)$. $a_{11}+a_{21}+a_{22}$ represents the total potential demand during the sales season. $b_{i}$ is channel $i$ 's marginal demand per price. $\theta$ is the sensitivity of switch-over towards price difference in the discount period.We assume that consumers make independent decisions in the two periods, and therefore do not consider the demands of period $2\left(D_{21}\right.$ and $\left.D_{22}\right)$ as a function of $p_{11}$.

The linear demand functions are adopted for two reasons. First, the linear structure well 
characterizes the demand diffusion across channels (Bass, 1969;Chiang, Chhajed, \& Hess, 2003), especially for the online channel in which product information is disseminated by consumers' comments (Duan, Gu, \& Whinston, 2008). Second, linear models are widely used in marketing and supply chain management literature due to their parsimony and tractability for analysis (Tang \& Yin, 2007; Liu, Anderson, \& Cruz, 2012). Also,the results derived from linear models can also be applied for other demand forms that can be approximated or locally approximated by linear functions.

\subsection{Model Assumptions}

Since the product will be sold with the traditional channel in both periods and with the online channel in the discount period, the retailer needs to set the optimal prices for the traditional channel dynamicallyin the two periods, and the price for the online channel in the discount period only. To properly solve the model, we have a number of assumptions.

Assumption 1. The pricesof the two channels satisfy $p_{11} \geq p_{21} \geq p_{22}>v$.

Assumption 2. The initial inventory $Q$ satisfies $a_{11}<Q<a_{11}+a_{21}+a_{22}$.

Assumption 2 ensures that there is leftover inventory at the end of Period 1; however, the initial inventoryQdoes not exceed the total potential demand of the two channels.

Assumption 3. Consumers' price sensitivitiesin the premium period and in the discount period satisfy $b_{1}<b_{2}$.

Assumption 3 reflects that consumers who purchase in the premium period are less sensitive to the price than those who purchase in the discount period.

Assumption 4. The potential demands of the two channelsin thetwo periods satisfy $a_{11}>a_{21}>a_{22}$.

Assumption 4 means that the potential demand in the premium period is larger than that in the discount period, and the potential demand of the traditional channel is larger than that of the online channel.

Without loss of generality, Assumptions 3 and 4 are introduced to simplify mathematical analyses. Subsection 6.4 explores the scenarios when Assumption 3 and 4 are violated. 


\section{Model Formulation and Solution}

In this section, we present basic models and their extensions. We first present the mode where the retailer only employs the traditional channel with dynamic pricing (i.e., Mode 1). Then we introduce the mode where the retailer introduces the online channel in the discount period (i.e., Mode 2). We compare the two basic models. We further incorporate the difference of return rate and service between the two channels in modeling.

\subsection{Basic Models}

\subsubsection{Mode 1:Traditional Channel Only with Dynamic Pricing in the Two Periods}

In this mode, we consider that the retailer only employsthe traditional channel for both periods with dynamic pricing. The total revenue for the retailer can be expressed as follows

$$
\pi\left(p_{11}, p_{21}\right)=p_{11}\left(a_{11}-b_{1} p_{11}\right)+p_{21}\left(a_{21}-b_{2} p_{21}\right)-v(Q-D)
$$

s.t. $D \leq Q$, where $D=a_{11}-b_{1} p_{11}+a_{21}-b_{2} p_{21}$.

The first two terms in the equation are the revenue of the traditional channel in two periods, respectively, and the third term is the loss of the remaining inventory at the end of the sales season. The retailer's objective is to maximize its revenue. With the Lagrangian multiplier, the optimization problem (1) faced by the retailercan be written as follows:

$\max \pi\left(p_{11}, p_{21}\right)=\max \left\{p_{11}\left(a_{11}-b_{1} p_{11}\right)+p_{12}\left(a_{21}-b_{2} p_{21}\right)-v(Q-D)+\lambda_{1}(Q-D)\right\}$, where $\lambda_{1} \geq 0, D=a_{11}-b_{1} p_{11}+a_{21}-b_{2} p_{21}$.

Theorem 1. When only the traditional channel is employed for both periods with dynamic pricing,

(1) If $Q>Q^{*}$, the optimal prices are

$$
\begin{aligned}
& p_{11}{ }^{*}=\frac{a_{11}-b_{1} v}{2 b_{1}} \\
& p_{21}{ }^{*}=\frac{a_{21}-b_{2} v}{2 b_{2}}
\end{aligned}
$$

(2) If $Q<Q^{*}$, the optimal prices are 


$$
\begin{aligned}
& p_{11}{ }^{*}=\frac{a_{11} b_{2}+\left(2 a_{11}+a_{21}-2 Q\right) b_{1}}{2 b_{1}\left(b_{1}+b_{2}\right)} \\
& {p_{21}}^{*}=\frac{a_{21} b_{1}+\left(a_{11}+2 a_{21}-2 Q\right) b_{2}}{2 b_{2}\left(b_{1}+b_{2}\right)},
\end{aligned}
$$

where $Q^{*}=\frac{1}{2}\left(a_{11}+a_{21}+v b_{1}+v b_{2}\right)$.

Proof. See the Appendix.

Q*representsa threshold point of the inventory,whichis determined by thetotal potential demand, unit clearing loss, and price sensitivities. It is derived based on the first-order condition that the marginal profit with respect to the change of prices $\left(p_{11}, p_{12}\right)$ equals zero because of the quasi-concavity of $\pi\left(p_{11}, p_{21}\right)$ holds. When $Q=Q^{*}$, the optimal prices in (1) and (2) are the same, and the demand just equals to inventory; when the inventory is larger than the threshold point, the retailer will have some unsold at the end of period 2 with optimal prices of Theorem 1(1), the unsold inventory will then be cleared with a salvage price; When the inventory is less than the threshold point, the retailer will sell out the entire inventory with optimal prices of Theorem 1(2).

Proposition 1. When only the traditional channel is employed for both periods with dynamic pricing,

(1) If $Q>Q^{*}, p_{11}^{*}$ increases with $a_{11}$, but keeps a constant with $a_{21} ; p_{21}^{*}$ increases with $a_{21}$, but keeps a constant with $a_{11}$;

(2) If $Q<Q^{*}, p_{11}^{*}$ increases with $a_{11}$ and $a_{21} ; p_{21}^{*}$ increases with $a_{11}$ and $a_{21}$; where $Q^{*}=\frac{1}{2}\left(a_{11}+a_{21}+v b_{1}+v b_{2}\right)$.

Proposition $1^{\dagger}$ suggests that if the inventory of the product is sufficiently large, then the price in a period only be affected by its own potential demand. But if the inventory is not very large, theprice increases with the potential demandsin both periods.

\subsubsection{Mode 2:OnlineChannel As a Supplement}

In this mode, the retailer introduces the online channel in the discount period to maximize its revenue. First, the retailer uses the traditional channel in the premium period, and then it employs both

\footnotetext{
${ }^{\dagger}$ Because the proof of most propositions is straightforward, we did not present it due to page limit.
} 
traditional and the online channels in the discount period. Thus the retailer's payoff includes the revenue from the traditional channel in the two periods and that from the online channel in the discount period. The total revenue for the retailer can be expressed as follows:

$$
\begin{aligned}
\pi\left(p_{11}, p_{21}, p_{22}\right)= & p_{11}\left(a_{11}-b_{1} p_{11}\right)+p_{21}\left[a_{21}-b_{2} p_{21}+\theta\left(p_{22}-p_{21}\right)\right] \\
& +p_{22}\left[a_{22}-b_{2} p_{22}+\theta\left(p_{21}-p_{22}\right)\right]-v(Q-D)
\end{aligned}
$$

s.t. $D \leq Q$, where $D=a_{11}-b_{1} p_{11}+a_{21}-b_{2} p_{21}+a_{22}-b_{2} p_{22}$ represents the total demand.

The first and second terms represent the revenue from the traditional channel in the two periods. The third term is the revenue from the online channel in the discount period. The last term is the loss of the remaining inventory at the end of the sales season.

With the Lagrangian multiplier, the optimizationproblem (2) can be written as follows:

$$
\begin{gathered}
\max \pi\left(p_{11}, p_{21}, p_{22}\right)=\max \left\{p_{11}\left(a_{11}-b_{1} p_{11}\right)+p_{21}\left[a_{21}-b_{2} p_{21}+\theta\left(p_{22}-p_{21}\right)\right]+p_{22}\left[a_{22}-b_{2} p_{22}+\theta\left(p_{21}-p_{22}\right)\right]\right. \\
\left.-v(Q-D)+\lambda_{1}(Q-D)\right\}
\end{gathered}
$$

where $\lambda_{1} \geq 0, D=a_{11}-b_{1} p_{11}+a_{21}-b_{2} p_{21}+a_{22}-b_{2} p_{22}$.

Theorem 2. When the retailer sells the product with the traditional channel in both periods and the online channel in the discount period,

(1) If $Q \geq Q^{*}$, the optimal prices are

$$
\begin{aligned}
& p_{11}{ }^{*}=\frac{a_{11}-v b_{1}}{2 b_{1}} \\
& p_{21}{ }^{*}=\frac{\theta\left(a_{21}+a_{22}\right)+b_{2} a_{21}-v b_{2}{ }^{2}-2 \theta v b_{2}}{2 b_{2}\left(b_{2}+2 \theta\right)} \\
& {p_{22}}^{*}=\frac{\theta\left(a_{21}+a_{22}\right)+b_{2} a_{22}-v b_{2}{ }^{2}-2 \theta v b_{2}}{2 b_{2}\left(b_{2}+2 \theta\right)}
\end{aligned}
$$

(2) If $Q<Q^{*}$, the optimal prices are

$$
\begin{aligned}
& p_{11}{ }^{*}=\frac{2 a_{11} b_{2}+b_{1}\left(2 a_{11}-2 Q+a_{21}+a_{22}\right)}{\left.2 b_{1} b_{1}+2 b_{2}\right)} \\
& p_{21}{ }^{*}=\frac{b_{2}^{2}\left[a_{11}+3 a_{21}+a_{22}-2 Q\right]+\theta b_{2}\left[2 a_{11}+4 a_{21}+4 a_{22}-4 Q\right]+\theta b_{1}\left(a_{21}+a_{22}\right)+b_{1} b_{2} a_{21}}{2 b_{2}\left(b_{2}+2 \theta\right)\left(b_{1}+2 b_{2}\right)} \\
& p_{22}{ }^{*}=\frac{b_{2}^{2}\left[a_{11}+3 a_{22}+a_{21}-2 Q\right]+\theta b_{2}\left[2 a_{11}+4 a_{21}+4 a_{22}-4 Q\right]+\theta b_{1}\left(a_{21}+a_{22}\right)+b_{1} b_{2} a_{22}}{2 b_{2}\left(b_{2}+2 \theta\right)\left(b_{1}+2 b_{2}\right)}
\end{aligned}
$$

where $Q^{*}=\frac{1}{2}\left(a_{11}+a_{21}+a_{22}+v b_{1}+2 v b_{2}\right)$.

Proof. See the Appendix.

Similar to Theorem $1, Q *$ is a function of the total potential demand, unit clearing loss, and price sensitivities. It is derived based on the first-order condition that the marginal profit 
with respect to the change of prices $\left(p_{11}, p_{12}, p_{22}\right)$ equals to zero. Substituting the optimal prices (Eq. (3) and (4))into the total demand expression, we can obtain that when $Q$ is not very large, the retailer chooses to sell out the entire inventory by adjusting the prices. Otherwise, the prices are constant with $Q$, and the retailer's revenue decreases with $Q$ when $Q$ exceeds a threshold point $Q^{*}$. Therefore, the optimal order quantity should be less than the threshold point. For a fixed inventory, the retailer tends to sell out the entire inventory when the potential demand or the unit loss incurred by the remaining inventory is large.

Proposition 2. When the retailer sells the product with the traditional channel in both periods and the online channel in the discount period,

(1) If $Q>\frac{1}{2}\left[a_{11}+a_{21}+a_{22}+v\left(b_{1}+2 b_{2}\right)\right]$, the optimal prices keep constant with $Q$;

(2) If $Q<\frac{1}{2}\left[a_{11}+a_{21}+a_{22}+v\left(b_{1}+2 b_{2}\right)\right]$, the optimal prices decrease with $Q$.

Proposition 3. When the retailer sells the product with the traditional channel in both periods and the online channel in the discount period,

(1) $p_{11}^{*}$ keeps constant with $\theta$;

(2) ${p_{21}}^{*}$ decreases and ${p_{22}}^{*}$ increases with $\theta$.

Proof. See the Appendix.

Proposition 3 suggests that the retailer should decrease the price difference between the online and traditional channels to increase the revenue as consumers' sensitivity of switch-over towards price difference increases.

Proposition 4.Regarding the price difference of the traditional channel in the two periods:

(1) Mode 2 (dual-channel) has a larger price difference between the two periods than Mode 1 (single-channel).

(2) Mode 2 has the same price as Mode 1 in the premium period, but it has a lower price than Mode 1 in the discount period when $Q>\frac{1}{2}\left[a_{11}+a_{21}+a_{22}+v\left(b_{1}+2 b_{2}\right)\right]$.

Proof.See the Appendix.Proposition 4 illustrates the price change of the traditional channel when the online channel is introduced in the discount period.

Proposition 5.When the retailer sells the product with the traditional channel in both periods and the online channel in the discount period, 
(1) If $Q>Q^{*}, p_{11}^{*}$ increases with $a_{11}$, but keeps a constant with $a_{21}$ and $a_{22} ; p_{21}^{*}$ and $p_{22}^{*}$ increases with $a_{21}$ and $a_{22}$, but keeps a constant with $a_{11}$;

(2)If $Q<Q^{*}, p_{11}^{*}, p_{21}^{*}$ and $p_{22}^{*}$ increases with $a_{11}, a_{21}$ and $a_{22}$;

where $Q^{*}=\frac{1}{2}\left(a_{11}+a_{21}+a_{22}+v b_{1}+2 v b_{2}\right)$.

Proposition 5 is in the same line as suggested in Proposition 1.

\subsection{Extended Models}

\subsubsection{Return Rate}

Due to asymmetric information, product returns occur when the product does not fit consumer preference. Compared with the traditional channel, the return rate of the online channel is much higher. According to Grimaldi(2013), the return rate of the traditional channel is about $8.7 \%$, while that of the online channel is $18 \%-35 \%$. Therefore, this subsection focuses on the effect of the online channel's return rate on pricing.

We assume that the return rate of the online channel is $\boldsymbol{\tau}$ in the discount period and the loss incurred by each returned product is denoted as $v_{1}$. For the convenience of analysis, we treat the return rate of the traditional channel to be zero. Please note that the analysis results can still be applied when the return rate of the traditional channel is non-zero, but the interpretation of $\tau$ will be the difference of the return rates between the two channels.

We now have the retailer's revenue function as follows:

$$
\begin{aligned}
& \pi\left(p_{11}, p_{21}, p_{22}\right)=p_{11}\left(a_{11}-b_{1} p_{11}\right)+p_{21}\left[a_{21}-b_{2} p_{21}+\theta\left(p_{22}-p_{21}\right)\right]+p_{22}\left[a_{22}-b_{2} p_{22}+\theta\left(p_{21}-p_{22}\right)\right] \\
& -\tau v_{1}\left[a_{22}-b_{2} p_{22}+\theta\left(p_{21}-p_{22}\right)\right]-v(Q-D) \\
& \text { s.t. } D \leq Q \text {, where } D=a_{11}-b_{1} p_{11}+a_{21}-b_{2} p_{21}+a_{22}-b_{2} p_{22} .
\end{aligned}
$$

The first and second terms are the revenue from the traditional channel in the two periods. The third term is the revenue from the online channel in the discount period. The fourth term is the loss due to the return, and the last term is the loss due to the leftoverinventory. With the Lagrangian multiplier, the optimizationproblem (5) can be written as follows: 


$$
\begin{aligned}
\max \pi\left(p_{11}, p_{21}, p_{22}\right)= & \max \left\{p_{11}\left(a_{11}-b_{1} p_{11}\right)+p_{21}\left[a_{21}-b_{2} p_{21}+\theta\left(p_{22}-p_{21}\right)\right]+p_{22}\left[a_{22}-b_{2} p_{22}+\theta\left(p_{21}-p_{22}\right)\right]\right. \\
& \left.-\tau v_{1}\left[a_{22}-b_{2} p_{22}+\theta\left(p_{21}-p_{22}\right)\right]-v(Q-D)+\lambda_{1}(Q-D)\right\}
\end{aligned}
$$

where $\lambda_{1} \geq 0$ and $D=a_{11}-b_{1} p_{11}+a_{21}-b_{2} p_{21}+a_{22}-b_{2} p_{22}$.

Theorem 3. Given the return rate of the online channel $\tau$,

(1) If $Q \geq Q^{*}$, the optimal prices are

$$
\begin{aligned}
& p_{11}{ }^{*}=\frac{a_{11}-v b_{1}}{2 b_{1}} \\
& {p_{21}}^{*}=\frac{b_{2} a_{21}+\theta\left(a_{21}+a_{22}\right)}{2 b_{2}\left(b_{2}+2 \theta\right)}-\frac{v}{2} \\
& {p_{22}}^{*}=\frac{\tau v_{1}-v}{2}+\frac{a_{21}+a_{22}}{4 b_{2}}-\frac{a_{21}-a_{22}}{4\left(b_{2}+2 \theta\right)} .
\end{aligned}
$$

(2) If $Q<Q^{*}$, the optimal prices are

$$
\begin{aligned}
& p_{11}^{*}=\frac{2 a_{11} b_{2}-b_{1} b_{2} \tau v_{1}+b_{1}\left(2 a_{11}-2 Q+a_{21}+a_{22}\right)}{2 b_{1}\left(b_{1}+2 b_{2}\right)} \\
& p_{21}{ }^{*}=\frac{b_{2}^{2}\left[a_{11}+3 a_{21}+a_{22}-2 Q-2 \theta \tau v_{1}-b_{2} \tau v_{1}\right]+\theta b_{2}\left[2 a_{11}+4 a_{21}+4 a_{22}-4 Q\right]+\theta b_{1}\left(a_{21}+a_{22}\right)+b_{1} b_{2} a_{21}}{2 b_{2}\left(b_{2}+2 \theta\right)\left(b_{1}+2 b_{2}\right)} \\
& p_{22}{ }^{*}=\frac{b_{2}^{2}\left[a_{11}+3 a_{22}+a_{21}-2 Q+2 \theta \tau v+b_{1} \tau v_{1}+b_{2} \tau v_{1}\right]+\theta b_{2}\left[2 a_{11}+4 a_{21}+4 a_{22}-4 Q+2 b_{1} \tau v_{1}\right]+\theta b_{1}\left(a_{21}+a_{22}\right)+b_{1} b_{2} a_{22}}{2 b_{2}\left(b_{2}+2 \theta\right)\left(b_{1}+2 b_{2}\right)},
\end{aligned}
$$

where $Q^{*}=\frac{1}{2}\left(a_{11}+a_{21}+a_{22}-\tau v_{1} b_{2}+v b_{1}+2 v b_{2}\right)$.

Proof. The proof is similar to Theorem 2.And the derivation of Q* is similar to that in Theorem 2.

Proposition 6. Giventhe return rate of the online channel $\tau$,

(1) If $Q \geq Q^{*}, p_{11}{ }^{*}, p_{21}{ }^{*}$ keep constant with $\tau$, but ${p_{22}}^{*}$ increases with $\tau$.

(2) If $Q<Q^{*},{p_{11}}^{*}, p_{21}{ }^{*}$ decrease with $\tau$, but $p_{22}^{*}$ increases with $\tau$.

Proposition 6means that the prices of the traditional channel are not affected by the return rate of the online channel when the inventory is less than the threshold point $Q^{*}$. Otherwise, the prices of the traditional channel decrease to induce more consumers to purchase the products via the traditional channel. And the threshold point of inventory, $Q^{*}$ decreases with $\tau$. From Theorem 3, we can see that the retailer tends to keep the remaining inventory when the return rate is large instead of selling out the entire inventory.

\subsubsection{Service Difference}

Service quality is an important factor affecting consumers' purchasing decisions. The 
traditional channel could offer customer services and experience that cannot be provided by the online channel. For example, consumers could try on shoes and clothes in abricks-and-mortar store, experiencing the product personally. Consumers may also enjoy after-sale services that are offered in a bricks-and-mortar store (Wagner, 2002). Consumers who value such services are more likely to purchase from the traditional channel (Yao \& Liu, 2005).

The demand function of the traditional channel in the premium period is $D_{11}=a_{11}-b_{1} p_{11}$. In the discount period, we assume that the demand function of the traditional channel is $D_{21}=a_{21}-b_{2} p_{21}+\theta_{1}\left(p_{22}-p_{21}\right)+\theta_{2} S$; the demand function of the online channel is $D_{22}=a_{22}-b_{2} p_{22}+\theta_{1}\left(p_{21}-p_{22}\right)-\theta_{2} S$, where $S$ represents the service difference, and $\theta_{2}$ represents consumers’ sensitivity of switch-over towards the service difference.

The model has two main features when we incorporate the service difference between the two channels. First, the demand switch-over caused by the price difference $\theta_{1}$ is different from the service difference $\theta_{2}$. The price difference between the traditional channel and the online channel is caused by not only service, but also channel convenient and consumers' acceptance of the online channel (Cattani, Perdikaki, \& Marucheck 2007;Fruchter \& Tapiero, 2005;Tsay \& Agrawal, 2004). To analyze the effect of the service difference on demand switch-over, the service difference is singled out. Second, please note that $D_{21}+D_{22}=a_{21}-b_{2} p_{21}+a_{22}-b_{2} p_{22}$, the total demand in Period 2 is affected only by prices but not by service. The service difference only affects demand switch-over between these two channels.Then we have the retailer's revenue function:

$$
\begin{aligned}
\pi\left(p_{11}, p_{21}, p_{22}\right)= & p_{11}\left(a_{11}-b_{1} p_{11}\right)+p_{21}\left[a_{21}-b_{2} p_{21}+\theta_{1}\left(p_{22}-p_{21}\right)+\theta_{2} S\right] \\
& +p_{22}\left[a_{22}-b_{2} p_{22}+\theta_{1}\left(p_{22}-p_{21}\right)-\theta_{2} S\right]-v\left(Q-D_{11}-D_{21}-D_{22}\right)
\end{aligned}
$$

s.t. $D_{11}+D_{21}+D_{22} \leq Q$.

The first and second terms are the traditional channel's revenue in the two periods. The third term is the online channel's revenue in the discount period. The last term is the loss due to the leftoverinventory.

Theorem 4. With the service difference between two channels,

(1) If $Q \geq Q^{*}$, the optimal prices are 


$$
\begin{aligned}
& p_{11}^{*}=\frac{a_{11}-v b_{1}}{2 b_{1}} \\
& {p_{21}}^{*}=\frac{\theta_{1}\left(a_{21}+a_{22}\right)-2 \theta_{1} v b_{2}-v b_{2}^{2}+b_{2}\left(a_{21}+\theta_{2} S\right)}{2 b_{2}\left(b_{2}+2 \theta_{1}\right)} \\
& {p_{22}}^{*}=\frac{\theta_{1}\left(a_{21}+a_{22}\right)-2 \theta_{1} v b_{2}-v b_{2}{ }^{2}+b_{2}\left(a_{22}-\theta_{2} S\right)}{2 b_{2}\left(b_{2}+2 \theta_{1}\right)}
\end{aligned}
$$

(2) If $Q<Q^{*}$, the optimal prices are,

$$
\begin{aligned}
& p_{11}{ }^{*}=\frac{2 a_{11} b_{2}+b_{1}\left(2 a_{11}-2 Q+a_{21}+a_{22}\right)}{\left.2 b_{1} b_{1}+2 b_{2}\right)} \\
& p_{21}{ }^{*}=\frac{b_{2}^{2}\left(a_{11}+3 a_{21}+a_{22}-2 Q\right)+\theta_{1} b_{2}\left[2 a_{11}+4\left(a_{21}+a_{22}-Q\right)\right]+\theta_{1} b_{1}\left(a_{21}+a_{22}\right)+b_{1} b_{2} a_{21}}{2 b_{2}\left(b_{2}+2 \theta_{1}\right)\left(b_{1}+2 b_{2}\right)}+\frac{S \theta_{2}}{2\left(b_{2}+2 \theta_{1}\right)} \\
& p_{22}{ }^{*}=\frac{b_{2}{ }^{2}\left(a_{11}+3 a_{22}+a_{21}-2 Q\right)+\theta_{1} b_{2}\left[2 a_{11}+4\left(a_{21}+a_{22}-Q\right)\right]+\theta_{1} b_{1}\left(a_{21}+a_{22}\right)+b_{1} b_{2} a_{22}}{2 b_{2}\left(b_{2}+2 \theta_{1}\right)\left(b_{1}+2 b_{2}\right)}-\frac{S \theta_{2}}{2\left(b_{2}+2 \theta_{1}\right)}
\end{aligned}
$$

where $Q^{*}=\frac{a_{11}+a_{21}+a_{22}+v\left(b_{1}+2 b_{2}\right)}{2}$.

Proof. The proof is similar to Theorem 2.

Proposition 7. With the service difference between two channels, $p_{11}{ }^{*}$ keeps constant with $S ; p_{21}{ }^{*}$

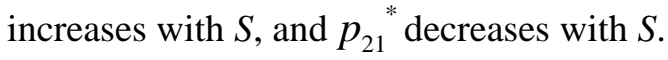

Proposition 8. With the service difference between two channels, $p_{11}{ }^{*}$ keeps constant with $\theta_{2} ; p_{21}{ }^{*}$ increases with, and ${p_{21}}^{*}$ decreases with $\theta_{2}$.

Propositions 7and 8show that the price of the traditional channel in the premium period is not affected by the service difference. And the price difference between the traditional and the online channels in the discountperiod decreases when consumers are sensitive to the service difference. The threshold point of inventory $\left(\frac{a_{11}+a_{21}+a_{22}+v\left(b_{1}+2 b_{2}\right)}{2}\right.$ in Theorem 4) keeps constant with $S$.

\section{The Retailer's Risk Attitude}

The demand of the online channel could beaffected by some non-product or service-related factors such as Internet accessibility and consumers' acceptance that may be difficult for the retailer to observe (Chiang, Chhajed, \& Hess, 2003). Therefore, in this subsection, we assume that the online channel demand is a random variable, and examine how the retailer's risk attitude affects itspricing strategy. Prior studies suggested risk attitude as an important factor affecting a firm's decision (Borgonovo \& Peccati, 2009).

We assume the demand function of the online channel in the discount period as: 


$$
D_{22}=\tilde{a}_{22}-b_{2} p_{22}+\theta\left(p_{21}-p_{22}\right),
$$

where $\tilde{a}_{22}$ is a random variable, it represents the potential demand of the online channel in Period 2.

We assume $\tilde{a}_{22}=a_{22}+\varepsilon$, where $a_{22}$ is the mean of the potential intrinsic demand, $\varepsilon$ follows a normal distribution, and $E(\varepsilon)=0, \operatorname{Var}(\varepsilon)=\sigma^{2}$.

Since the online demand in the discount period is stochastic, we should consider the risk attitude of the retailer intoitspricing decision. The preference theory provides a framework for incorporating decision makers' risk propensity in their decision process. The valuation measure is known in the preference theory as "certainty equivalent". It is defined as a certain value for an uncertain event which a decision maker is willing to accept (Raiffa, 1968;Holloway, 1979). One form of the utility function dominant in both theoretical and applied work in areas of decision theory and finance is the exponential utility function $U(\pi)=-\exp (-\pi / R)$, where $R$ is the retailer's risk tolerance level and $\pi$ is revenue. A value of $R<\infty$ implies risk-averse behavior. When $R$ approaches $\infty$, risk-neutral behavior is implied (Howard, 1988). Correspondingly, the certainty equivalent of $\pi$ in the basic model is expressed by the following equation: $E(U)=E(\pi)-\frac{\operatorname{Var}(\pi)}{2 R}$, where $R$ is the retailer's risk tolerance level, $\operatorname{Var}(\pi)$ is the variance of revenue $\pi$. We have $\operatorname{Var}(\pi)=\left(p_{22}+v\right)^{2} \sigma^{2}$, when $Q>D ; \operatorname{Var}(\pi)=p_{22}{ }^{2} \sigma^{2}$, when $Q \leq D$. Therefore, the optimization problem is to maximize the expected utility $E(U)$, as follows:

$$
E(U)=p_{11}\left(a_{11}-b_{1} p_{11}\right)+p_{21}\left[a_{21}-b_{2} p_{21}+\theta\left(p_{22}-p_{21}\right)\right]+p_{22}\left[a_{22}-b_{2} p_{22}+\theta\left(p_{21}-p_{22}\right)\right]-v(Q-D)-\frac{\operatorname{Var}(\pi)}{2 R}
$$

s.t. $D \leq Q$,where $D=a_{11}-b_{1} p_{11}+a_{21}-b_{2} p_{21}+a_{22}-b_{2} p_{22}$ represents the total demand.

Theorem 5. When the retailer sells the product with the traditional channel in both periods and the online channel in the discount period,

(1) If $Q \geq Q^{* \prime}$, the optimal prices are 


$$
\begin{aligned}
& p_{11}^{*}=\frac{a_{11}-v b_{1}}{2 b_{1}} \\
& p_{21}{ }^{*}=\frac{\theta\left(a_{21}+a_{22}\right) R+b_{2} a_{21} R-v b_{2}{ }^{2} R-2 \theta v b_{2} R}{2 b_{2}\left(b_{2}+2 \theta\right) R+\sigma^{2}\left(b_{2}+\theta\right)}+\frac{\sigma^{2}\left(a_{21}-2 \theta v-b_{2} v\right)}{4 b_{2}\left(b_{2}+2 \theta\right) R+2 \sigma^{2}\left(b_{2}+\theta\right)}{ }^{\prime} \\
& p_{22}^{*}=\frac{\left[\theta\left(a_{21}+a_{22}\right)+b_{2} a_{22}\right] R-\sigma^{2} v\left(\theta+b_{2}\right) / 2}{2 b_{2}\left(b_{2}+2 \theta\right) R+\sigma^{2}\left(b_{2}+\theta\right)}-\frac{v}{2}
\end{aligned}
$$

(2) If $Q<Q^{* \prime}$, the optimal prices are

$$
\begin{aligned}
& p_{11}^{*}=\frac{b_{2}{ }^{2}\left[a_{11} \sigma^{2}+4 R a_{11}\left(b_{1}+b_{2}+2 \theta\right)+2 R b_{1}\left(-2 Q+a_{21}+a_{22}\right)\right]+\sigma^{2}\left[b_{1} b_{2}\left(-2 Q+2 a_{11}+a_{21}+2 a_{22}\right)+2 b_{1} \theta\left(-Q+a_{11}+a_{21}+a_{22}\right)\right]+4 R b_{1} b_{2} \theta\left(-2 Q+2 a_{11}+a_{21}+a_{22}\right)}{2 b_{1}\left[2 b_{2}{ }^{2} R\left(2 b_{2}+b_{1}+4 \theta\right)+\sigma^{2}\left(b_{1} b_{2}+b_{1} \theta+b_{2}{ }^{2}\right)+4 R b_{1} b_{2} \theta\right]} \\
& p_{21}{ }^{*}=\frac{b_{2}\left[\left(\sigma^{2}+4 R \theta\right)\left(a_{11}+2 a_{21}+2 a_{22}-2 Q\right)+2 R a_{21} b_{1}\right]+2 b_{2}{ }^{2} R\left(a_{11}+3 a_{21}+a_{22}-2 Q\right)+2 R b_{1} \theta\left(a_{21}+a_{22}\right)+a_{21} b_{1} \sigma^{2}}{2\left[2 R b_{2}{ }^{2}\left(2 b_{2}+b_{1}+4 \theta\right)+\sigma^{2}\left(b_{2}{ }^{2}+b_{1} b_{2}+b_{1} \theta\right)+4 R b_{1} b_{2} \theta\right]} \\
& p_{22}{ }^{*}=\frac{b_{2}{ }^{2} R\left(a_{11}+3 a_{22}+a_{21}-2 Q\right)+2 b_{2} R \theta\left(a_{11}+2 a_{21}+2 a_{22}-2 Q\right)+R b_{1} \theta\left(a_{21}+a_{22}\right)+a_{22} b_{1} b_{2} R}{2 R b_{2}{ }^{2}\left(2 b_{2}+b_{1}+4 \theta\right)+\sigma^{2}\left(b_{2}{ }^{2}+b_{1} b_{2}+b_{1} \theta\right)+4 R b_{1} b_{2} \theta}
\end{aligned}
$$

where

$Q^{* \prime}=\frac{2 R b_{2}\left(b_{2}+2 \theta\right)\left(a_{11}+a_{21}+a_{22}+b_{1} v+2 b_{2} v\right)+\sigma^{2}\left[\left(a_{11}+a_{21}+a_{22}\right)\left(b_{2}+\theta\right)+b_{2} v\left(b_{1}+4 \theta+3 b_{2}\right)+\theta\left(b_{1} v+a_{21}+a_{22}\right)\right]}{2\left[2 R b_{2}\left(b_{2}+2 \theta\right)+\sigma^{2}\left(b_{2}+\theta\right)\right]}$.

Proof. See the Appendix.

Given the complexity of analytical expressions given in Theorem 5, we will use a numerical example to explore some properties of the optimal solutions. Let $\sigma=10, Q=850, a_{11}=600$, $a_{21}=520 \quad, \quad a_{22}=280 \quad, \quad b_{1}=1 \quad, \quad b_{2}=2 \quad, \quad \theta=4 \quad, \quad v=20 \quad, \quad$ and $R \in[20,40,60,80,100,120,140,160,180,200,220]$. From Fig. 2, we can see that the optimal prices are determined by Theorem 5(2) when $R \leq 140$; and the optimal prices are determined by Theorem 5(1) when $R \geq 160$.

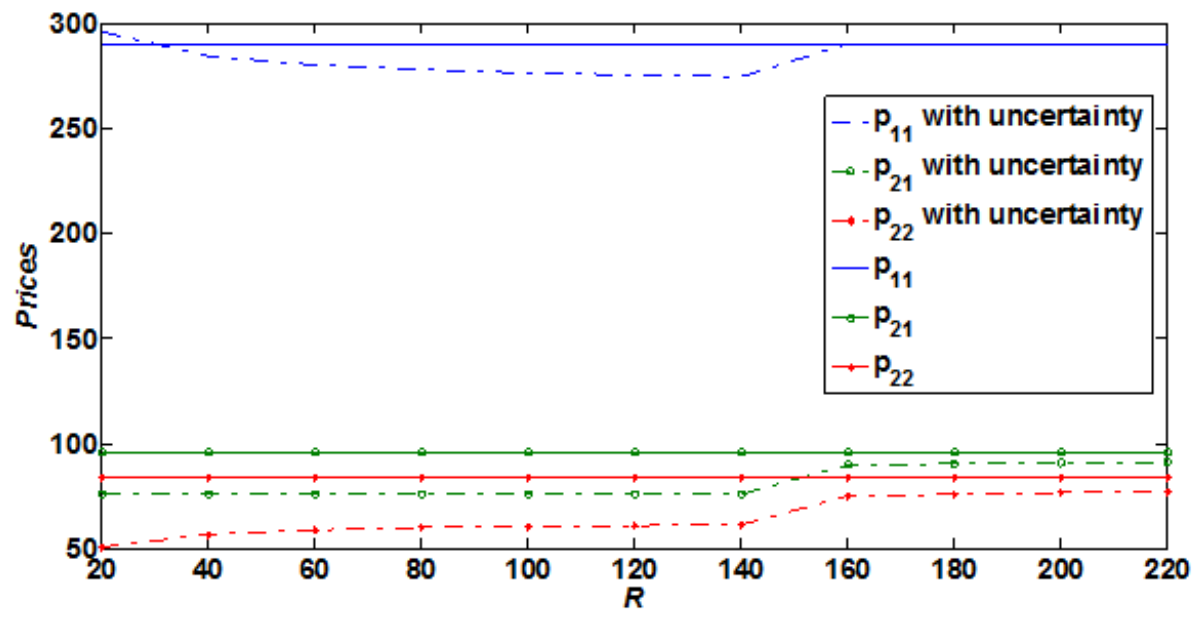

Fig.2 Channel price as a function of $R$

The prices of the two channels in the discount perioddecrease with the presence of demand uncertainty of theonline channel, but increase with the retailer's risk tolerance level. When the retailer 
is much risk averse ( $R \leq 140$ ), it prefers to set lower prices in both periods than those without demand uncertainty. As its risk tolerance increases ( $R \geq 160)$, it tends to set higher prices to obtain the maximum revenueratherthan selling out the entire inventory.

Specifically, when the retailer is extremely risk averse (when $R<30$ ), the price of the traditional channel in the premium period is higher and the price of the online channel in the discount period is lower than that without demand uncertainty. In other words, the retailer will keep the traditional channel profitable, butreduceits online price to deal with online demand uncertainty. When the retailer's risk tolerance increases ( $40 \leq R \leq 140$ ), the retailer adjusts both the premiumprice and the discount price of the two channels to deal with demand uncertainty. If the retailer's risk tolerance is very high ( $R \geq 160$ ), it only changes prices in the discount period to deal with online demanduncertainty.

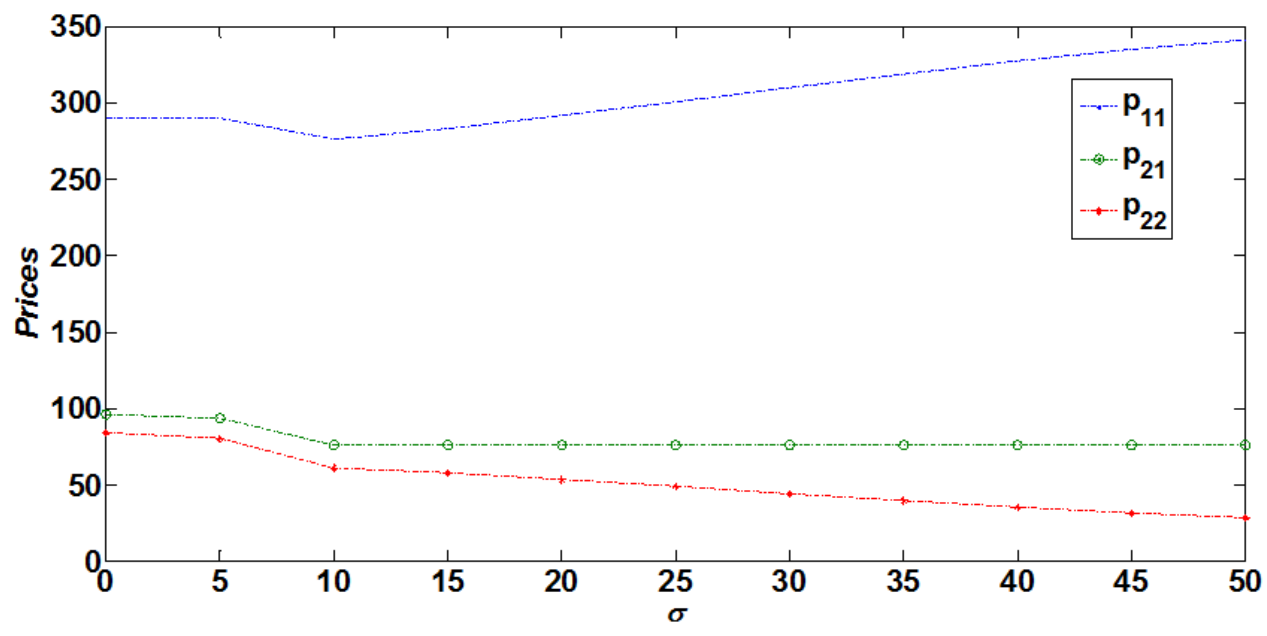

Fig.3 Channel price as a function of the standard deviation $\sigma$

Fig.3 presentshow the prices change with the standard deviation of online demand. When the standard deviation is small ( $0 \leq \sigma \leq 10$ in this numerical example), the premium and the discount prices of both channels $\left(p_{11}, p_{21}, p_{22}\right)$ decrease with the standard deviation.In other words, the retailer decreases the premium and the discount prices to deal with the increasinguncertaintyin this situation. When the standard deviation of the online demand is large ( $10 \leq \sigma \leq 50)$,the discount price of the online channel $\left(p_{22}\right)$ decreases, the premium price of the traditional channel $\left(p_{11}\right)$ increases, and the discount price of thetraditional channel $\left(p_{21}\right)$ keeps constant. In other words, the retailer increases the premium price of the traditional channel and decreases the discount price of the online channeltodeal with the increasing uncertaintyin this situation. 
The revenue change with demand uncertainty is given in Appendix Figs.A1 and A2. The expected revenue with demand uncertainty is lower than the revenue with deterministic demand, and the expected revenue increases with the retailer's risk tolerance but decreases with the standard deviation.

\section{Numerical Examples}

In this section, we explore some scenarios to verify the results obtained in the above sections and offer more insights. The general model parameters are set as follows: in the premium period the potential demand $a_{11}=600$, and the price sensitivity $b_{1}=1$. In the discount period, the potential demand of the traditional and the online channels are $a_{21}=520$ and $a_{22}=280$, respectively, and the price sensitivityis $b_{2}=2$. The sensitivity of switch-over towards price difference is $\theta=4$. The unit loss caused by the remaining inventory is $v=20$. These values satisfy Assumptions 2, 3, and 4 .

\subsection{Comparison of Channel Selection Solutions}

\subsubsection{Comparison of Single Channel and Dual Channels}

This subsection focuses on analyzing the effect of the online channel's potential demand on product prices and retail revenue with the two basic modes mentioned in Section 4.1. The parameters are set as follows: $Q=800, a_{22} \in[0,50,100,150,200,250,300]$. When $a_{22}=0$, it is equivalent to Mode 1.

Table 1 Comparison of Optimal Solutions

\begin{tabular}{|c|c|c|c|c|c|c|c|}
\hline & $p_{11}$ & $p_{21}$ & $p_{22}$ & $D_{11}$ & $D_{21}$ & $D_{22}$ & $\pi$ \\
\hline Single Channel & 290 & 120 & -- & 310 & 280 & -- & 119300 \\
\hline \multicolumn{8}{|l|}{ Dual-Channel $a_{22}$} \\
\hline 50 & 290 & 70 & 50 & 310 & 280 & 40 & 109270 \\
\hline 100 & 290 & 78 & 57 & 310 & 280 & 70 & 112930 \\
\hline 150 & 290 & 80 & 60 & 310 & 280 & 100 & 116970 \\
\hline 200 & 290 & 88 & 72 & 310 & 280 & 120 & 121380 \\
\hline 250 & 290 & 90 & 80 & 310 & 280 & 140 & 126170 \\
\hline 300 & 290 & 98 & 87 & 310 & 280 & 170 & 131330 \\
\hline
\end{tabular}

From Table 1, we can see that introducing the online channel in the discount period could decrease the leftover inventory, but it also makes the price of the traditional channel lower in the 
discount period. The results indicate the occurrence of channel conflict. If the potential demand of the online channel is small, the retailer has less revenue with dual channels than with single channel. If the potential demand of the online channel is large, the retailer has more revenue with dual channels. The price of the traditional channel in the discount period increases with the potential demand of the online channel. Therefore, introducing the online channel could decrease the unsold inventory effectively. However, whether the dual-channel mode benefits the retailer depends on the potential demand of the online channel.

\subsubsection{Comparison of Partial and Complete Dual Channels}

This subsectionexamines the scenario in which the online channel is introduced in both periods (complete dual channels). We compare the optimal prices of this scenario with those of the scenario whenthe online channel only introduced in the discount period (partial dual channels) as we discussed above.We assume that the potential demand of the online channel in the premium period, $a_{12}=300$, other parameters are the same as in the general setting.

Table 2Optimal Prices and Revenueswith Partial Dual Channels

\begin{tabular}{llllllll}
\hline$Q$ & $p_{11}$ & $p_{21}$ & $p_{22}$ & $D_{11}$ & $D_{21}$ & $D_{22}$ & $\pi$ \\
\hline 600 & 320 & 126 & 114 & 280 & 220 & 100 & 128720 \\
550 & 330 & 136 & 124 & 270 & 200 & 80 & 126220 \\
500 & 340 & 146 & 134 & 260 & 180 & 60 & 122720 \\
450 & 350 & 156 & 144 & 250 & 160 & 40 & 118220 \\
400 & 360 & 166 & 154 & 240 & 140 & 20 & 112720 \\
350 & 370 & 176 & 164 & 230 & 120 & 0 & 106220 \\
300 & 380 & 186 & 174 & 220 & 100 & 0 & 98720 \\
\hline
\end{tabular}

Table 30ptimal Prices and RevenueswithComplete Dual Channels

\begin{tabular}{llllllllll}
\hline$Q$ & $p_{11}$ & $p_{12}$ & $p_{21}$ & $p_{22}$ & $D_{11}$ & $D_{12}$ & $D_{21}$ & $D_{22}$ & $\pi$ \\
\hline 600 & 275 & 258 & 148 & 136 & 258 & 108 & 177 & 57 & 132800 \\
550 & 283 & 267 & 156 & 144 & 250 & 100 & 160 & 40 & 128220 \\
500 & 292 & 275 & 164 & 152 & 242 & 92 & 143 & 23 & 122800 \\
450 & 300 & 283 & 173 & 161 & 233 & 83 & 127 & 7 & 116550 \\
400 & 308 & 292 & 181 & 169 & 225 & 75 & 110 & 0 & 109470 \\
350 & 317 & 300 & 189 & 177 & 217 & 67 & 93 & 0 & 101550 \\
300 & 325 & 308 & 198 & 186 & 208 & 58 & 77 & 0 & 92803 \\
\hline
\end{tabular}

The results in Tables 2 and 3 suggest that the complete-dual-channels is profitable when inventory is sufficiently large. When the inventory is not very large, the partialdualchannels are more 
profitable. If the inventory is small (i.e., $Q<400$ ) the single-channel solution is more profitable than other channel solutions. Introducing the online channel in the premium period decreases the premium price of the traditional channel but increases the prices of both channels in the discount period. The results suggest channel conflict. When $Q=400$, the two channels only co-exist in Period 1 in Table 3 . By comparing Tables 2 and 3, we can see that introducing the online channel in the discount period (Table 2) results in more revenue than using the online channel in the premium period (Table 3).

In summary, the retailer shall adopt the traditional channel alone when the inventory level is very small. When the inventory is large, the retailer could introduce the online channel in the discount period. When the inventory is extremely large, the retailer will benefit from introducing the two channels in both periods. The results are summarized in Table $4 . Q_{i}(i=1,2)$ in Table 4 are thresholds determined by the channel potential demand $a_{i j}(i, j=1,2)$, the marginal demand per price $b_{i}(i=1,2)$, the sensitivity of switch-over towards price difference $\theta_{i}(i=1,2)$, and the unit loss of the product incurred by clearing $v$.

Table 4 Optimal Channels Choices with Inventory Level $Q$

\begin{tabular}{lll}
\hline \multicolumn{1}{c}{ Inventory level $Q$} & \multicolumn{1}{c}{ Premium period } & \multicolumn{1}{c}{ Discount period } \\
\hline$Q<Q_{1}$ & Traditional channel & Traditional channel \\
$Q_{1}<Q<Q_{2}$ & Traditional channel & Traditional +online channels \\
$Q>Q_{2}$ & Traditional +online channels & Traditional +online channels \\
\hline
\end{tabular}

\subsection{Channel Conflict}

This subsection focuses on the effect of dynamic pricing on channel conflict. We present following three scenarios: (1) the traditional channel employs static pricingin the twoperiods (i.e., single-static); (2) extending scenario 1, we introduce the online channel in the discount period (i.e., dual-static); (3) the traditional channel employs dynamic pricing in the two periods, and the online channel is introduced in the discount period (i.e., dual-dynamic). The inventory $Q \in[600,800]$ and other parameters are the same as in the general setting.

Figs.4and 5show that when the inventory exceeds a certain threshold point $Q^{*}$, the prices and the revenues tend to be fixed. In the scenario of the single channel with static pricing, the threshold point $Q^{*}<600$. In other scenarios the threshold point $Q^{*}=700$. In addition, Fig.4indicates that the premium price with the dual-static mode is lower than that that withthe single-static mode. That is, introducing the online channel decreases the price of the traditional channel when the retailer employs 
static pricing for the traditional channel; however, when the retailer employs dynamic pricing for the traditional channel, the premium price with the dual-dynamic mode is much larger than that with the dual-static mode and the single-static mode, and the discount price is much lower than that with the dual-static mode. By implementing dynamic pricing, the price difference becomes larger, and retailer's revenue increases compared with static price (Fig. 5).

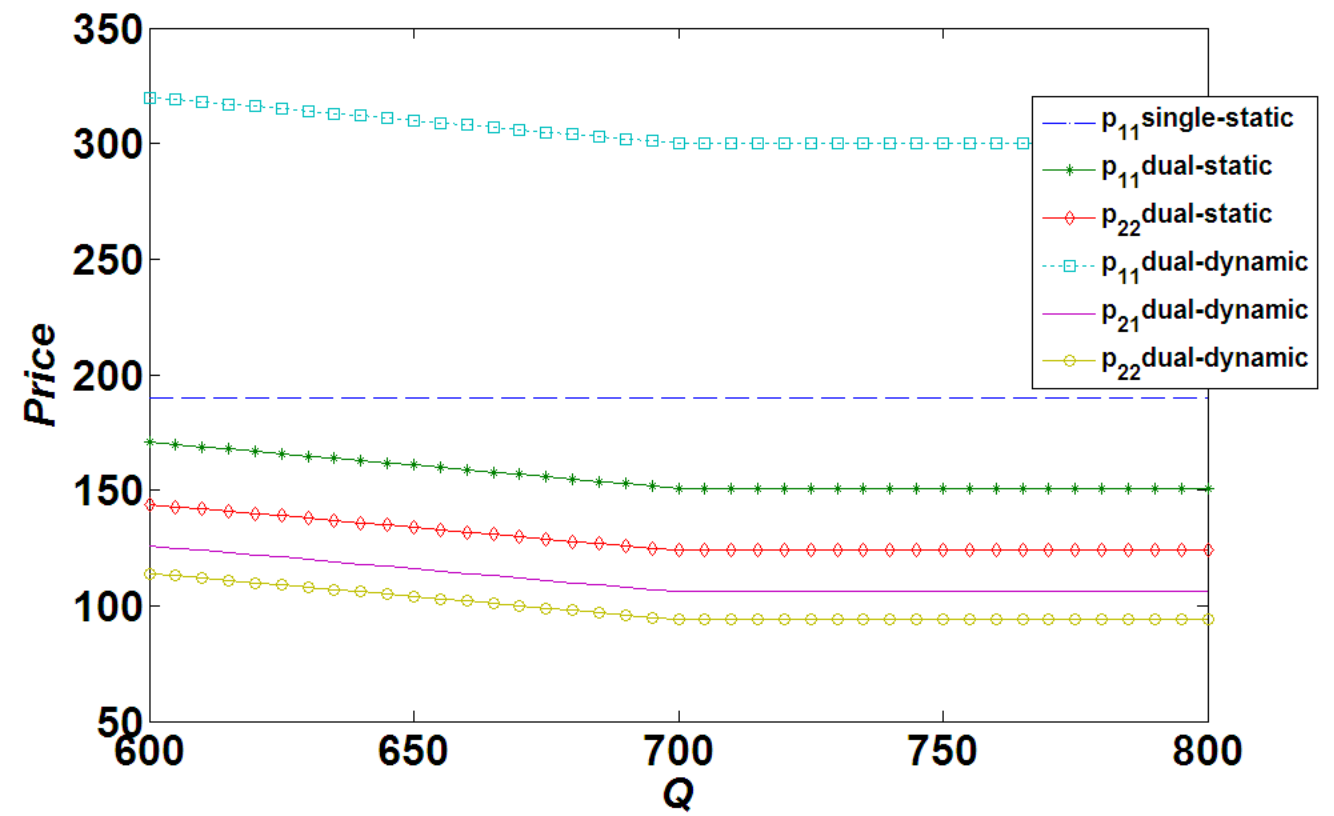

Fig.4Channel price as a function of $Q$

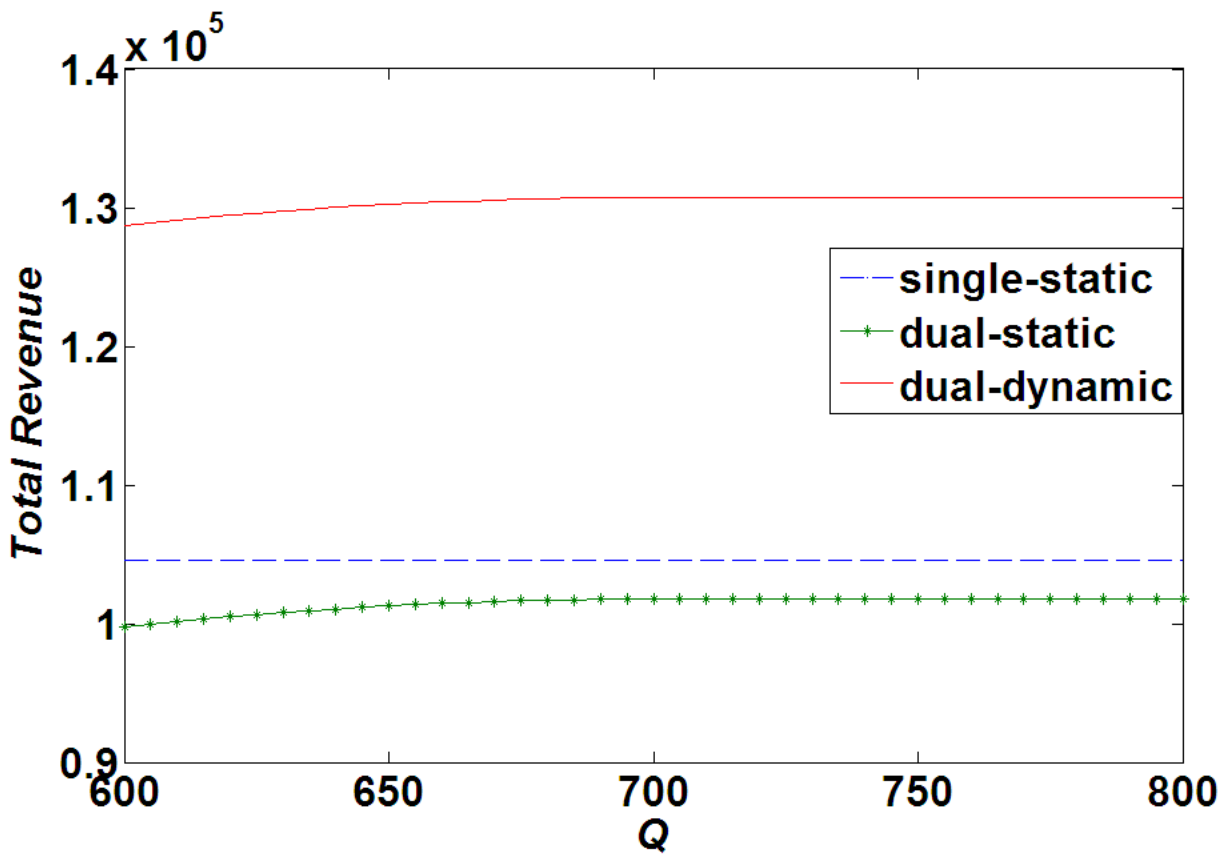

Fig.5Total revenue as a function of $Q$

Fig. 5 shows the total revenue decreases after introducing the online channel when static pricing is used for the traditional channel. Figs. A3 and A4 in Appendix A illustrate the change of the 
revenues of the two channels respectively. The results suggest the existence of channel conflict. But when dynamic pricing is used for the traditional channel, both the revenue of the traditional channel and the total revenue increase. In other words, dynamic pricing mitigates channel conflict.

\subsection{Assumption Violation}

This subsection explores the changes of optimal solutions when Assumptions 3 and 4 are not satisfied.

\section{Example 1}

In this example, we present the change of optimal solutions when Assumption 4 is violated which assumes that the potential demands in the two periods with two channels satisfy $a_{11}>a_{21}>a_{22}$.Similar to Yao \& Liu (2005), assuming the total potential demand in the discount period is fixed, we discuss how the prices change with the proportion of the online demand.Let $\alpha$ denote the proportion of the online demand in period 2. The total potential demands of the traditional channel and the online channel in the discount period are $a_{21}+a_{22}=600$. When $\alpha<0.5$, $a_{11}>a_{21}>a_{22}$; or else $a_{11}>a_{22}>a_{21}$. Other parameters are set as follows: $Q=700, a_{11}=600, b_{1}=1, b_{2}=2, \theta=4$, and $v=20$. Table 5shows that prices of the traditional channel decrease, while the price of online channel increases, when the potential demand of online channel increases. The total revenue reaches the minimum when $\alpha=0.5$. In other words, channel conflict is at its maximum when the potential demands of the two channels are equal.

Table 50ptimal Prices and Revenues

\begin{tabular}{llllllll}
\hline$\alpha$ & $p_{11}$ & $p_{21}$ & $p_{22}$ & $D_{11}$ & $D_{21}$ & $D_{22}$ & Revenue \\
\hline 0.1 & 290 & 77 & 53 & 310 & 290 & 50 & 113880 \\
0.2 & 290 & 74 & 56 & 310 & 260 & 80 & 112620 \\
0.3 & 290 & 71 & 59 & 310 & 230 & 110 & 111720 \\
0.4 & 290 & 68 & 62 & 310 & 200 & 140 & 111180 \\
0.5 & 290 & 65 & 65 & 310 & 170 & 170 & 111000 \\
0.6 & 290 & 62 & 68 & 310 & 140 & 200 & 111180 \\
0.7 & 290 & 59 & 71 & 310 & 110 & 230 & 111720 \\
0.8 & 290 & 56 & 74 & 310 & 80 & 260 & 112620 \\
0.9 & 290 & 53 & 77 & 310 & 50 & 290 & 113880 \\
\hline
\end{tabular}




\section{Example 2}

In this example, we present the change of optimal solutions when Assumption 3 is violated which assumes consumers' price sensitivities in the premium period and in the discount period satisfy $b_{1}<b_{2}$. Let $b_{1}=1$, and $b_{2} \in[0.5,1.5]$.Consumers become more sensitive to price as $b_{2}$ increases. Other parameters are set as follows: $a_{21}=520, a_{22}=280, \theta=4$ and $v=20$. Table 6suggests that the prices of the two channels and sales revenues decrease as $b_{2}$ increases. When the price sensitivity in Period 1 is much larger than that in Period $2\left(b_{1}>b_{2} \in[0.5,0.6]\right)$, the prices in Period 1 are lower than that in period 2. In other words, Period 1 is the discount period and Period 2 becomes the premium period.However, $p_{21}$ is smaller than $p_{11}$ when $b_{2} \in[0.7,1)$.This is because the prices are not only affected byprice sensitivities, but also affected by the potential demands in the two periods. When the difference of price sensitivities in the two periods is small and the total potential demand in Period 2 is larger than that in Period 1, the price in Period 2 with small price sensitivity may be even lower than that in Period 1 with large price sensitivity.

Table 6Optimal Prices and Revenues

\begin{tabular}{llllllll}
\hline$b_{2}$ & $p_{11}$ & $p_{21}$ & $p_{22}$ & $D_{11}$ & $D_{21}$ & $D_{22}$ & Revenue \\
\hline 0.5 & 300 & 407 & 393 & 300 & 260 & 140 & 250850 \\
0.6 & 300 & 340 & 326 & 300 & 260 & 140 & 224170 \\
0.7 & 300 & 293 & 279 & 300 & 260 & 140 & 205110 \\
0.8 & 300 & 257 & 243 & 300 & 260 & 140 & 190820 \\
0.9 & 300 & 229 & 215 & 300 & 260 & 140 & 179700 \\
1 & 300 & 207 & 193 & 300 & 260 & 140 & 170800 \\
1.1 & 300 & 188 & 175 & 300 & 260 & 140 & 163520 \\
1.2 & 300 & 173 & 160 & 300 & 260 & 140 & 157450 \\
1.3 & 300 & 160 & 147 & 300 & 260 & 140 & 152310 \\
1.4 & 300 & 149 & 136 & 300 & 260 & 140 & 147910 \\
1.5 & 300 & 140 & 127 & 300 & 260 & 140 & 144090 \\
\hline
\end{tabular}

\section{Example 3}

This example examines the change of optimal solutions when both Assumption 3 and 4 are violated. Assume that the potential demand, $a_{11}=280, a_{21}=520, a_{22}=600, b_{1}=2$, and $b_{2}=1$. Similar to Example 2, Period 2 becomes the premium period with higher price and strong demand, and Period 1 is the discount period. In Period 2 (the premium period), both channels are used. And the 
online channel is the main channel. Andin Period 1 (the discount period) only the traditional channel is used. Thus this case is totally different from the modeling background we discussed in this paper. It could be corresponding to when the retailer sells the product whose value increasesovertime on the market, such as when selling the air tickets, and the retailer mainly uses the online channel to reach consumers.

From Table 7, we can see that the optimal prices in Period 2 are higher than that of Period 1. And the price of the online channel is higher than that of the traditional channel which is due to a large potential demand of the online channel. The prices in the two periods decrease with the inventory level $Q$. The prices of the traditional channel in the two periods are identical when the inventory exceeds a threshold point. In other words, when the supplement channel (in this case, the traditional channel) is introduced in bothperiods and the inventory is very large, the prices of the supplement channel in bothperiods should keep constant.

Table 70ptimal Prices and Revenues

\begin{tabular}{llllllll}
\hline$Q$ & $p_{11}$ & $p_{21}$ & $p_{22}$ & $D_{11}$ & $D_{21}$ & $D_{22}$ & Revenue \\
\hline 700 & 140 & 210 & 210 & 0 & 330 & 370 & 147090 \\
750 & 140 & 180 & 190 & 0 & 360 & 390 & 138840 \\
800 & 140 & 140 & 160 & 0 & 380 & 420 & 128090 \\
850 & 131 & 131 & 157 & 18 & 494 & 338 & 120164 \\
900 & 118 & 118 & 145 & 43 & 507 & 350 & 115789 \\
950 & 106 & 106 & 132 & 68 & 519 & 363 & 110164 \\
1000 & 93 & 93 & 120 & 93 & 532 & 375 & 103289 \\
\hline
\end{tabular}

\section{Discussion and Conclusion}

The online channel plays different roles for different industries. This study focuses on the sales of a short-life-cycle product as in the apparel industry using the online channels as a supplement to the traditional channel. We consider a retailer can employboth the traditional and the online channels, but mostly relies on the traditional channel. We explore such a channel mix: the retailer uses the traditional channel in the premium period, and both channels in the discount period.

The use of dynamic pricing for the traditional channel captures the nature of short-life cycle products. Such a strategy is consistent with prior studies about perishable products (Zhao \& Zheng, 2000;Aviv \& Pazgal, 2008). Further, the dynamic pricing mechanism can also mitigate channel conflict effectively. If the retailer uses static pricing for the traditional channel in the two periods, then sales revenue will decrease when the online channel is introduced in Period 2 (Fig.5and Figs.A3and 
A4in Appendix).

Our results also suggest that price and service differences between these two channels will not change the optimalprice in the premium period when the online channel is introduced in the discount period. When the product inventory is small, the optimal price in the premium period increases (Theorems 1-4). In other words, the introduction of the online channel in the discount period will not decrease the premium price of the traditional channel, but rather increase the premium price when the inventory is not very large. Thus, choosing the right time to introduce the online channel could improve the retailer's revenue and mitigate channel conflict.

The study further shows that whether dual channels benefit the retailer is contingent on the potential demand of the online channel and the product inventory (Tables 1-3). The retailer should adopt single channel when the demand of the online channel or the inventory is very small. Otherwise, the retailer will benefit from using both channels. And the online channel should be added first in the discount period, and then expanded to the premium periodif needed.

The retailer's expected revenue with demand uncertainty is lower than the revenue with deterministic demand. The revenue increases with retailer's risk tolerance but decreases with the standard deviationof the online channel demand (Appendix Figs.A1 and A2). The retailer adjusts thepremium price and the online channel price to sell out the entire inventory when it is risk-averse. The optimal pricesin bothperiodsdecrease with the retailer's risk tolerance. When the retailer's risk tolerance level approaches infinite,only prices in the discount perioddecrease to deal with onlineuncertainty (Theorem 5 and Fig.2).

In summary, there are three significant implications for practitioners. First, the retailer could mitigate channel conflict by choosing the right time to introduce the online channel. Secondly, the retailer could mitigate channel conflict by differentiating the prices between the two channels. Third, introducing the online channel could effectively reduce the unsold inventory. However, whether the dual-channel solution is beneficial to the retailer depends on the potential demand of the online channel and the product inventory.

A limitation of this studyis that it assumes the demands inthe two periodsareindependent. There is a possibility that the lower premium price will exhaust more demand in the premium period, and decreasethe demand in the discount period. When the premium price is very high, customers may delay their purchase to the discount period. Hence the potential demand in the discount period 
increases. If we model the potential demand in period 2 is affected by the premium price: $D_{21}=a_{21}-b_{2} p_{21}+\theta\left(p_{22}-p_{21}\right)+k l p_{11}, \quad D_{22}=a_{22}-b_{2} p_{22}+\theta\left(p_{21}-p_{22}\right)+k(1-l) p_{11}, \quad D_{11}=a_{11}-b_{1} p_{11}$ , where $k$ represents the demand switch-over toward price from period 1 to period 2 , and $k<b_{1}$, then the total demand of the two periods is $D=a_{11}+a_{21}+a_{22}-\left(b_{1}-k\right) p_{11}-b_{2} p_{21}-b_{2} p_{22}$. Because of mathematical complexity, we are not able to give analytical solutions. Given that the total demand in the basic model is $D=a_{11}+a_{21}+a_{22}-b_{1} p_{11}-b_{2} p_{21}-b_{2} p_{22}$, and that in the new model is $D=a_{11}+a_{21}+a_{22}-\left(b_{1}-k\right) p_{11}-b_{2} p_{21}-b_{2} p_{22}$, we cannot perform meaningful comparisons between the optimal solutions of the two models,so future studies could investigate this direction.

As consumers often compare utility before deciding where to purchase, future studies could investigate the effect of consumers' strategic choices on channel conflict and dynamic pricing. In addition, since the apparel industry suffers from the problem of maintaining high inventory, future research could explore the optimal inventory when the retailer employs dual channels. Further, as the model parameters may vary from industry to industry, it would be a good idea to obtain more accurate and realistic parameter values to give better empirical implications.

\section{Acknowledgments}

We thank the review panel for their comments that have significantly improved the lucidity of paper. This work was supported by theNational Nature Science Foundation of China [grant numbers 71602103]; the Shandong Normal University [grant numbers 304132 and 15SQR017]; andthe National Science Foundation [grant numbers 1420758]. The usual disclaimer applies.

\section{Reference}

Aviv, Y., \& Pazgal, A. (2008). Optimal pricing of seasonal products in the presence of forward-looking consumers. M\&Som-Manufacturing \& Service Operations Management, 10(3), 339-359. doi: DOI 10.1287/msom.1070.0183

Bass, F. M. (1969). A new product growth model for consumer durables. Management Science, 15(5), 215-227.

Borgonovo, E., \& Peccati, L. (2009). Financial management in inventory problems: Risk averse vs 
risk neutral policies. International Journal of Production Economics, 118(1), 233-242.

Cachon, G. P., \& Swinney, R. (2009). Purchasing, Pricing, and Quick Response in the Presence of Strategic Consumers. Management Science, 55(3), 497-511. doi: DOI 10.1287/mnsc.1080.0948

Cai, G., Zhang, Z. G., \& Zhang, M. (2009). Game theoretical perspectives on dual-channel supply chain competition with price the discount $\mathrm{s}$ and pricing schemes. International Journal of Production Economics, 117(1), 80-96.

Cao, J., So, K. C., \& Yin, S. (2016). Impact of an “online-to-store” channel on demand allocation, pricing and profitability. European Journal of Operational Research, 248(1), 234-245.

Carrillo, J. E., Vakharia, A. J., \& Wang, R. (2014). Environmental implications for online retailing. European Journal of Operational Research, 239(3), 744-755.

Cattani, K., Perdikaki, O., \& Marucheck, A. (2007). The perishability of online grocers. Decision Sciences, 38(2), 329-355. doi: DOI 10.1111/j.1540-5915.2007.00161.x

Chellappa, R. K., Sin, R. G., \& Siddarth, S. (2011). Price formats as a source of price dispersion: A study of online and offline prices in the domestic US airline markets. Information Systems Research, 22(1), 83-98.

Chen, J., \& Bell, P. C. (2012). Implementing market segmentation using full-refund and no-refund customer returns policies in a dual-channel supply chain structure. International Journal of Production Economics, 136(1), 56-66. doi: DOI 10.1016/j.ijpe.2011.09.009

Chiang, W. Y. K., Chhajed, D., \& Hess, J. D. (2003). Direct-marketing, indirect profits: A strategic analysis of dual-channel supply-chain design. Management Science, 49(1), 1-20. doi: DOI 10.1287/mnsc.49.1.1.12749

Chuang, C. H., Wang, C. X., \& Zhao, Y. B. (2014). Closed-loop supply chain models for a high-tech product under alternative reverse channel and collection cost structures. International Journal Of Production Economics, 156, 108-123. doi: DOI 10.1016/j.ijpe.2014.05.008

Dan, B., Xu, G. Y., \& Liu, C. (2012). Pricing policies in a dual-channel supply chain with retail services. International Journal Of Production Economics, 139(1), 312-320. doi: DOI 10.1016/j.ijpe.2012.05.014

Duan, W. J., Gu, B., \& Whinston, A. B. (2008). The dynamics of online word-of-mouth and product sales-An empirical investigation of the movie industry. Journal of retailing, 84(2), 233-242.

Dumrongsiri, A., Fan, M., Jain, A., \& Moinzadeh, K. (2008). A supply chain model with direct and 
retail channels. European Journal Of Operational Research, 187(3), 691-718. doi: DOI 10.1016/j.ejor.2006.05.044

Feng, Y., \& Gallego, G. (1995). Optimal starting times for end of season sales and optimal stopping times for promotional fares. . Management Science 41(8), 1371-1391.

Fruchter, G. E., \& Tapiero, C. S. (2005). Dynamic online and offline and offline channel pricing for heterogeneous customers in virtual acceptance. International Game Theory Review, 7(2), 137-150.

Gallego, G., \& Van Ryzin, G. J. (1994). Optimal dynamic pricing of inventories with stochastic demand over finite horizons. Management Science 40(8), 999-1020.

Grimaldi, P. (2013). Day of rejects: store and clerks gear up for gift exchanges Retrieved 05/03, 2013,

from http://www.projo.com/seasonal/holidays/content/BZ_RETURNS_1226_12-26-08_88CO6AO_ V22.314fb6b.html

Holloway, C. A. (1979). Decision Making Under Uncertainty: Models and Choices. New Jersey: Prentice-Hall.

Howard, R. A. (1988). Decision analysis: practice and promise. Management Science, 34(6), 679-695.

Johansen, J., \& Riis, J. O. (1995). Managing Seasonal Fluctuations In Demand - Practice And Experience Of Selected Industrial-Enterprises. Production Planning \& Control, 6(5), 461-468. doi: Doi 10.1080/09537289508930304

Kurata, H., Yao, D. Q., \& Liu, J. J. (2007). Pricing policies under direct vs. indirect channel competition and national vs. store brand competition. European Journal of Operational Research, 180(1), 262-281.

Li, Y. J., Wei, C. S., \& Cai, X. Q. (2012). Optimal pricing and order policies with B2B product returns for fashion products. International Journal of Production Economics, 135(2), 637-646.

Liu, Z. G., Anderson, T. D., \& Cruz, J. M. (2012). Consumer environmental awareness and competition in two-stage supply chains. European Journal Of Operational Research, 218(3), 602-613.

Liu, K., Zhou, Y., \& Zhang, Z. (2010). Capacitated location model with online demand pooling in a multi-channel supply chain. European Journal of Operational Research, 207(1), 218-231. 
Marom, O., \& Seidmann, A. (2011). Using “last-minute”sales for vertical differentiation on the Internet. Decision Support Systems, 51(4), 894-903.

Ofek, E., Katona, Z., \& Sarvary, M. (2011). Bricks-and-clicks: The impact of product returns on the strategies of multi-channel retailers. Marketing Science, 30(1), 42-60.

Park, S. Y., \& Keh, H. T. (2003). Modeling hybrid distribution channels:Game-theoretic analysis. Journal of Retailing and Consumer Services, 10(3), 155-167.

Raiffa, H. (1968). Decision Analysis: Introductory Lectures on Choices Under Uncertainty., Reading, MA. Addison-Wesley.

Riggins, F. J. (2004). A multichannel model of separating equilibrium in the face of the digital divide. Journal Of Management Information Systems, 21(2), 161-179.

Tang, C. S., \& Yin, R. (2007). Joint ordering and pricing strategies for managing substitutable products. Production And Operations Management, 16(1), 138-153.

Tedeschi, B. (2000, March 27). Compressed data; big companies go slowly in devising net strategy. New York Times.

Tsay, A. A., \& Agrawal, N. (2004). Channel conflict and coordination in the E-commerce age. Production and Operations Management, 13(1), 93-110.

Wagner, M. (2002). IT vendors embrace channel partners. B to B, 87(9), 1-2.

Yan, R. L., \& Pei, Z. (2009). Retail services and firm profit in a dual-channel market. Journal of Retailing and Consumer Services, 16(4), 306-314.

Yao, D. Q., \& Liu, J. J. (2005). Competitive pricing of mixed retail and e-tail distribution channels. Omega-International Journal Of Management Science, 33(3), 235-247. doi: DOI 10.1016/j.omega.2004.04.007

Yue, X. H., \& Liu, J. (2006). Demand forecast sharing in a dual-channel supply chain. European Journal Of Operational Research, 174(1), 646-667. doi: DOI 10.1016/j.ejor.2004.12.020

Zhao, W., \& Zheng, Y. S. (2000). Optimal dynamic pricing for perishable assets with nonhomogeneous demand. Management Science, 46(3), 375-388. doi: DOI 10.1287/mnsc.46.3.375.12063

\section{Appendix}




\section{Proof of Theorem 1.}

$\max \pi\left(p_{11}, p_{21}\right)=\max \left\{p_{11}\left(a_{11}-b_{1} p_{11}\right)+p_{12}\left(a_{21}-b_{2} p_{21}\right)-v(Q-D)+\lambda_{1}(Q-D)\right\}$

where $\lambda_{1} \geq 0, D=a_{11}-b_{1} p_{11}+a_{21}-b_{2} p_{21}$.

(1)when $\lambda_{1}=0$, which implies $Q \geq D$, the optimization problem is

$$
\max \pi\left(p_{11}, p_{21}\right)=\max \left\{p_{11}\left(a_{11}-b_{1} p_{11}\right)+p_{12}\left(a_{21}-b_{2} p_{21}\right)-v(Q-D)\right\}
$$

with Assumptions 1-4, we consider the first order conditions,

$$
\begin{aligned}
& \frac{\partial \pi}{\partial p_{11}}=a_{11}-2 b_{1} p_{11}-b_{1} v=0 \\
& \frac{\partial \pi}{\partial p_{21}}=a_{21}-2 b_{2} p_{21}-b_{2} v=0
\end{aligned}
$$

then obtain the optimal prices

$$
\begin{aligned}
& p_{11}{ }^{*}=\frac{a_{11}-v b_{1}}{2 b_{1}}, \\
& {p_{21}}^{*}=\frac{a_{21}-v b_{2}}{2 b_{2}},
\end{aligned}
$$

Substituting $p_{11}^{*}, p_{21}^{*}$ into $D$,

$D=a_{11}-b_{1} p_{11}^{*}+a_{21}-b_{2} p_{21}^{*}=a_{11}-\frac{b_{1}\left(a_{11}-v b_{1}\right)}{2 b_{1}}+a_{21}-\frac{b_{2}\left(a_{21}-v b_{2}\right)}{2 b_{2}}=\frac{a_{11}+a_{21}+v b_{1}+v b_{2}}{2}$ then we have $D=\frac{1}{2}\left(a_{11}+a_{21}+v b_{1}+v b_{2}\right)$. In this case, $Q \geq D$, that is, $Q \geq Q^{*}$, where $Q^{*}=\frac{1}{2}\left(a_{11}+a_{21}+v b_{1}+v b_{2}\right)$.

(2) when $\lambda_{1}>0$, which implies $Q=D$, then the optimization problem is

$$
\max \pi\left(p_{11}, p_{21}\right)=\max \left\{p_{11}\left(a_{11}-b_{1} p_{11}\right)+p_{12}\left(a_{21}-b_{2} p_{21}\right)-v(Q-D)+\lambda_{1}(Q-D)\right\} \text { with }
$$

Assumptions 1-4, we consider the first order conditions, 


$$
\begin{aligned}
& \frac{\partial \pi}{\partial p_{11}}=a_{11}+b_{1} \lambda_{1}-2 b_{1} p_{11}-b_{1} v=0, \\
& \frac{\partial \pi}{\partial p_{21}}=a_{21}+b_{2} \lambda_{1}-2 b_{2} p_{21}-b_{2} v=0 \\
& \frac{\partial \pi}{\partial \lambda_{1}}=Q-a_{11}-a_{21}+b_{1} p_{11}+b_{2} p_{21}=0
\end{aligned}
$$

then obtain the optimal solutions

$$
\begin{aligned}
& p_{11}{ }^{*}=\frac{a_{11} b_{2}+\left(2 a_{11}+a_{21}-2 Q\right) b_{1}}{2 b_{1}\left(b_{1}+b_{2}\right)} \\
& {p_{21}}^{*}=\frac{a_{21} b_{1}+\left(a_{11}+2 a_{21}-2 Q\right) b_{2}}{2 b_{2}\left(b_{1}+b_{2}\right)} . \\
& \lambda_{1}=\frac{a_{11}+a_{21}-2 Q}{b_{1}+2 b_{2}}
\end{aligned}
$$

And $\lambda_{1}=\frac{a_{11}+a_{21}-2 Q}{b_{1}+2 b_{2}}>0$ is equivalent to $Q<\frac{1}{2}\left(a_{11}+a_{12}+v b_{1}+v b_{2}\right)$, that is $Q<Q^{*}$, where $Q^{*}=\frac{1}{2}\left(a_{11}+a_{21}+v b_{1}+v b_{2}\right)$.

\section{Proof of Theorem 2.}

$$
\begin{array}{r}
\max \pi\left(p_{11}, p_{21}, p_{22}\right)=\max \left\{p_{11}\left(a_{11}-b_{1} p_{11}\right)+p_{21}\left[a_{21}-b_{2} p_{21}+\theta\left(p_{22}-p_{21}\right)\right]\right. \\
+p_{22}\left[a_{22}-b_{2} p_{22}+\theta\left(p_{21}-p_{22}\right)\right]-v(Q-D)+\lambda_{1}(Q-D)
\end{array}
$$

where $\lambda_{1} \geq 0, D=a_{11}-b_{1} p_{11}+a_{21}-b_{2} p_{21}+a_{22}-b_{2} p_{22}$.

(1) when $\lambda_{1}=0$, which implies $Q \geq D$, the optimization problem is

$$
\begin{gathered}
\max \pi\left(p_{11}, p_{21}, p_{22}\right)=\max \left\{p_{11}\left(a_{11}-b_{1} p_{11}\right)+p_{21}\left[a_{21}-b_{2} p_{21}+\theta\left(p_{22}-p_{21}\right)\right]\right. \\
+p_{22}\left[a_{22}-b_{2} p_{22}+\theta\left(p_{21}-p_{22}\right)\right]-v(Q-D)
\end{gathered}
$$

With the first order conditions, $\frac{\partial \pi}{\partial p_{11}}=0, \frac{\partial \pi}{\partial p_{21}}=0, \frac{\partial \pi}{\partial p_{22}}=0$, using Matlab 6.5, we obtain the optimal prices, 


$$
\begin{aligned}
& p_{11}{ }^{*}=\frac{a_{11}-v b_{1}}{2 b_{1}} \\
& p_{21}{ }^{*}=\frac{\theta\left(a_{21}+a_{22}\right)+b_{2} a_{21}-v b_{2}{ }^{2}-2 \theta v b_{2}}{2 b_{2}\left(b_{2}+2 \theta\right)}, \\
& {p_{22}}^{*}=\frac{\theta\left(a_{21}+a_{22}\right)+b_{2} a_{22}-v b_{2}{ }^{2}-2 \theta v b_{2}}{2 b_{2}\left(b_{2}+2 \theta\right)}
\end{aligned}
$$

Substituting $p_{11}{ }^{*}, p_{12}{ }^{*}, p_{22}{ }^{*}$ into $D_{11}, D_{12}, D_{22}$, we have $D=D_{11}+D_{21}+D_{22}$

$$
\begin{aligned}
& =a_{11}-b_{1} p_{11}^{*}+a_{21}-b_{2} p_{21}^{*}+\theta\left(p_{22}^{*}-p_{21}^{*}\right)+a_{22}-b_{2} p_{22}^{*}+\theta\left(p_{21}^{*}-p_{22}^{*}\right) \\
& =\frac{1}{2}\left[a_{11}+a_{21}+a_{22}+v\left(b_{1}+2 b_{2}\right)\right]
\end{aligned}
$$

The restriction condition $Q \geq D$ is equivalent to $Q \geq Q^{*}$, where $Q^{*}=\frac{1}{2}\left[a_{11}+a_{21}+a_{22}+v\left(b_{1}+2 b_{2}\right)\right]$.

(2) When $\lambda_{1}>0$, which implies $Q=D$, thenthe optimization problem is

$$
\begin{array}{r}
\max \pi\left(p_{11}, p_{21}, p_{22}\right)=\max \left\{p_{11}\left(a_{11}-b_{1} p_{11}\right)+p_{21}\left[a_{21}-b_{2} p_{21}+\theta\left(p_{22}-p_{21}\right)\right]\right. \\
+p_{22}\left[a_{22}-b_{2} p_{22}+\theta\left(p_{21}-p_{22}\right)\right]-v(Q-D)+\lambda_{1}(Q-D)
\end{array}
$$

Let $\frac{\partial \pi}{\partial p_{11}}=0, \frac{\partial \pi}{\partial p_{21}}=0, \frac{\partial \pi}{\partial p_{22}}=0, \frac{\partial \pi}{\partial \lambda_{1}}=0$, using Matlab 6.5, the optimal solutions are

$$
\begin{aligned}
& p_{11}^{*}=\frac{2 a_{11} b_{2}+b_{1}\left(2 a_{11}-2 Q+a_{21}+a_{22}\right)}{2 b_{1}\left(b_{1}+2 b_{2}\right)}, \\
& p_{21}{ }^{*}=\frac{b_{2}^{2}\left[a_{11}+3 a_{21}+a_{22}-2 Q\right]+\theta b_{2}\left[2 a_{11}+4 a_{21}+4 a_{22}-4 Q\right]+\theta b_{1}\left(a_{21}+a_{22}\right)+b_{1} b_{2} a_{21}}{2 b_{2}\left(b_{2}+2 \theta\right)\left(b_{1}+2 b_{2}\right)}, \\
& {p_{22}}^{*}=\frac{b_{2}^{2}\left[a_{11}+3 a_{22}+a_{21}-2 Q\right]+\theta b_{2}\left[2 a_{11}+4 a_{21}+4 a_{22}-4 Q\right]+\theta b_{1}\left(a_{21}+a_{22}\right)+b_{1} b_{2} a_{22}}{2 b_{2}\left(b_{2}+2 \theta\right)\left(b_{1}+2 b_{2}\right)} \\
& \lambda_{1}=\frac{a_{11}+a_{21}+a_{22}-2 Q+b_{1} v+2 b_{2} v}{b_{1}+2 b_{2}}
\end{aligned}
$$

Because $\lambda_{1}>0$, it is equivalent to $Q<Q^{*}$, where

$$
Q^{*}=\frac{1}{2}\left[a_{11}+a_{21}+a_{22}+v\left(b_{1}+2 b_{2}\right)\right]
$$

\section{Proof of Proposition 3.}


If $Q>\frac{1}{2}\left[a_{11}+a_{21}+a_{22}+v\left(b_{1}+2 b_{2}\right)\right]$

$\frac{\partial p_{11}^{*}}{\partial \theta}=0, \frac{\partial p_{21}{ }^{*}}{\partial \theta}=\frac{-\left(a_{21}-a_{22}\right)}{4 b_{2}^{2}\left(b_{2}+2 \theta\right)^{2}}, \frac{\partial p_{22}{ }^{*}}{\partial \theta}=\frac{a_{21}-a_{22}}{4 b_{2}^{2}\left(b_{2}+2 \theta\right)^{2}}$.

If $Q<\frac{1}{2}\left[a_{11}+a_{21}+a_{22}+v\left(b_{1}+2 b_{2}\right)\right]$, the derivatives are the same. From Assumption 4, $a_{21}>a_{22}$, then we can prove Proposition 3.

\section{Proof of Proposition 4.}

(1) From Theorem1, we can get the price difference of the traditional channel in Mode 1

is $\Delta_{1}={p_{11}}^{*}-{p_{21}}^{*}=\frac{a_{11} b_{2}-a_{21} b_{1}}{2 b_{1} b_{2}}$ for both $Q \geq Q^{*}$ and $Q<Q^{*}$;

From Theorem 2, we can get the price difference of the traditional channel in Mode 2 is

$\Delta_{2}=p_{11}{ }^{*}-p_{21}{ }^{*}=\frac{a_{11} b_{2}\left(b_{2}+2 \theta\right)-a_{21} b_{1} b_{2}-\left(a_{21}+a_{22}\right) b_{1} \theta}{2 b_{1} b_{2}\left(2 \theta+b_{2}\right)}$ for both $Q \geq Q^{*}$ and $Q<Q^{*}$;

then $\Delta_{1}-\Delta_{2}=-\frac{\left(a_{21}-a_{22}\right) \theta}{2 b_{2}\left(b_{2}+2 \theta\right)}<0$,

because $a_{21}>a_{22}$, hence Proposition 4(1) is obtained.

(2) It is obvious that the premium price are equal when

$$
Q>\frac{1}{2}\left[a_{11}+a_{21}+a_{22}+v\left(b_{1}+2 b_{2}\right)\right] ;
$$

and when $Q>\frac{1}{2}\left[a_{11}+a_{21}+a_{22}+v\left(b_{1}+2 b_{2}\right)\right], p_{21}^{*}{ }_{(\text {Mode } 2)}-p_{21(\text { Mode })}^{*}=-\frac{\left(a_{21}-a_{22}\right) \theta}{2 b_{2}\left(b_{2}+2 \theta\right)}<0$,

hence Proposition 4(2) is obtained.

Proof of Theorem 5. 
The optimization problem is as follows:

$$
\begin{aligned}
\max E(U)= & \max \left\{p_{11}\left(a_{11}-b_{1} p_{11}\right)+p_{21}\left[a_{21}-b_{2} p_{21}+\theta\left(p_{22}-p_{21}\right)\right]\right. \\
& \left.+p_{22}\left[a_{22}-b_{2} p_{22}+\theta\left(p_{21}-p_{22}\right)\right]-v(Q-D)-\frac{\operatorname{Var}(\pi)}{2 R}\right\}
\end{aligned}
$$

s.t. $D \leq Q, \quad D=a_{11}-b_{1} p_{11}+a_{21}-b_{2} p_{21}+a_{22}-b_{2} p_{22}$.

Using Lagrangian multiplier,

$$
\begin{aligned}
& \max E(U)=\max \left\{p_{11}\left(a_{11}-b_{1} p_{11}\right)+p_{21}\left[a_{21}-b_{2} p_{21}+\theta\left(p_{22}-p_{21}\right)\right]\right. \\
& \left.\quad+p_{22}\left[a_{22}-b_{2} p_{22}+\theta\left(p_{21}-p_{22}\right)\right]-v(Q-D)-\frac{\operatorname{Var}(\pi)}{2 R}+\lambda_{1}(Q-D)\right\}
\end{aligned}
$$

(1) When $\lambda_{1}=0$, which implies $Q \geq D$, the optimization problem is

$$
\begin{aligned}
& \max E(U)=\max \left\{p_{11}\left(a_{11}-b_{1} p_{11}\right)+p_{21}\left[a_{21}-b_{2} p_{21}+\theta\left(p_{22}-p_{21}\right)\right]\right. \\
& \left.\quad+p_{22}\left[a_{22}-b_{2} p_{22}+\theta\left(p_{21}-p_{22}\right)\right]-v(Q-D)-\frac{\left(p_{22} \sigma+v \sigma\right)^{2}}{2 R}\right\}
\end{aligned}
$$

With the first order conditions $\frac{\partial \pi}{\partial p_{11}}=0, \frac{\partial \pi}{\partial p_{21}}=0, \frac{\partial \pi}{\partial p_{22}}=0$, using Matlab 6.5, we obtain the optimal prices,

$$
\begin{aligned}
& p_{11}{ }^{*}=\frac{a_{11}-v b_{1}}{2 b_{1}} \\
& p_{21}{ }^{*}=\frac{\theta\left(a_{21}+a_{22}\right) R+b_{2} a_{21} R-v b_{2}{ }^{2} R-2 \theta v b_{2} R}{2 b_{2}\left(b_{2}+2 \theta\right) R+\sigma^{2}\left(b_{2}+\theta\right)}+\frac{\sigma^{2}\left(a_{21}-2 \theta v-b_{2} v\right)}{4 b_{2}\left(b_{2}+2 \theta\right) R+2 \sigma^{2}\left(b_{2}+\theta\right)} ; \\
& p_{22}{ }^{*}=\frac{\left[\theta\left(a_{21}+a_{22}\right)+b_{2} a_{22}\right] R-\sigma^{2} v\left(\theta+b_{2}\right) / 2}{2 b_{2}\left(b_{2}+2 \theta\right) R+\sigma^{2}\left(b_{2}+\theta\right)}-\frac{v}{2}
\end{aligned}
$$

Substituting $p_{11}{ }^{*}, p_{12}{ }^{*}, p_{22}{ }^{*}$ into $D_{11}, D_{12}, D_{22}$, we have

$$
\begin{aligned}
& D=D_{11}+D_{21}+D_{22}=a_{11}-b_{1} p_{11}^{*}+a_{21}-b_{2} p_{21}^{*}+\theta\left(p_{22}^{*}-p_{21}^{*}\right)+a_{22}-b_{2} p_{22}^{*}+\theta\left(p_{21}^{*}-p_{22}^{*}\right)=Q^{* \prime} \text {, where } \\
& Q^{* \prime}=\frac{2 R b_{2}\left(b_{2}+2 \theta\right)\left(a_{11}+a_{21}+a_{22}+b_{1} v+2 b_{2} v\right)+\sigma^{2}\left[\left(a_{11}+a_{21}+a_{22}\right)\left(b_{2}+\theta\right)+b_{2} v\left(b_{1}+4 \theta+3 b_{2}\right)+\theta\left(b_{1} v+a_{21}+a_{22}\right)\right]}{2\left[2 R b_{2}\left(b_{2}+2 \theta\right)+\sigma^{2}\left(b_{2}+\theta\right)\right]} .
\end{aligned}
$$

The restriction condition $Q \geq D$ is equivalent to $Q \geq Q^{* \prime}$.

(2) When $\lambda_{1}>0$, which implies $Q=D$, the problem can be described as follows: 
$\max E(U)=\max \left\{p_{11}\left(a_{11}-b_{1} p_{11}\right)+p_{21}\left[a_{21}-b_{2} p_{21}+\theta\left(p_{22}-p_{21}\right)\right]\right.$

$\left.+p_{22}\left[a_{22}-b_{2} p_{22}+\theta\left(p_{21}-p_{22}\right)\right]-v(Q-D)-\frac{p_{22}{ }^{2} \sigma^{2}}{2 R}+\lambda_{1}(Q-D)\right\}$,

With the first order conditions, $\frac{\partial \pi}{\partial p_{11}}=0, \frac{\partial \pi}{\partial p_{21}}=0, \frac{\partial \pi}{\partial p_{22}}=0, \frac{\partial \pi}{\partial \lambda_{1}}=0$, we can obtain the optimal prices using Matlab 6.5,

$p_{11}{ }^{*}=\frac{b_{2}{ }^{2}\left[a_{11} \sigma^{2}+4 R a_{11}\left(b_{1}+b_{2}+2 \theta\right)+2 R b_{1}\left(-2 Q+a_{21}+a_{22}\right)\right]+\sigma^{2}\left[b_{1} b_{2}\left(-2 Q+2 a_{11}+a_{21}+2 a_{22}\right)+2 b_{1} \theta\left(-Q+a_{11}+a_{21}+a_{22}\right)\right]+4 R b_{1} b_{2} \theta\left(-2 Q+2 a_{11}+a_{21}+a_{22}\right)}{2 b_{1}\left[2 b_{2}{ }^{2} R\left(2 b_{2}+b_{1}+4 \theta\right)+\sigma^{2}\left(b_{1} b_{2}+b_{1} \theta+b_{2}{ }^{2}\right)+4 R b_{1} b_{2} \theta\right]}$
$p_{21}{ }^{*}=\frac{b_{2}\left[\left(\sigma^{2}+4 R \theta\right)\left(a_{11}+2 a_{21}+2 a_{22}-2 Q\right)+2 R a_{21} b_{1}\right]+2 b_{2}{ }^{2} R\left(a_{11}+3 a_{21}+a_{22}-2 Q\right)+2 R b_{1} \theta\left(a_{21}+a_{22}\right)+a_{21} b_{1} \sigma^{2}}{2\left[2 R b_{2}{ }^{2}\left(2 b_{2}+b_{1}+4 \theta\right)+\sigma^{2}\left(b_{2}{ }^{2}+b_{1} b_{2}+b_{1} \theta\right)+4 R b_{1} b_{2} \theta\right]}$
$p_{22}{ }^{*}=\frac{b_{2}{ }^{2} R\left(a_{11}+3 a_{22}+a_{21}-2 Q\right)+2 b_{2} R \theta\left(a_{11}+2 a_{21}+2 a_{22}-2 Q\right)+R b_{1} \theta\left(a_{21}+a_{22}\right)+a_{22} b_{1} b_{2} R}{2 R b_{2}{ }^{2}\left(2 b_{2}+b_{1}+4 \theta\right)+\sigma^{2}\left(b_{2}{ }^{2}+b_{1} b_{2}+b_{1} \theta\right)+4 R b_{1} b_{2} \theta}$

similar to Theorem 1,2, $\lambda_{1}>0$ is equivalent to $Q \leq Q^{* \prime}$ where $Q^{* \prime}=\frac{2 R b_{2}\left(b_{2}+2 \theta\right)\left(a_{11}+a_{21}+a_{22}+b_{1} v+2 b_{2} v\right)+\sigma^{2}\left[\left(a_{11}+a_{21}+a_{22}\right)\left(b_{2}+\theta\right)+b_{2} v\left(b_{1}+4 \theta+3 b_{2}\right)+\theta\left(b_{1} v+a_{21}+a_{22}\right)\right]}{2\left[2 R b_{2}\left(b_{2}+2 \theta\right)+\sigma^{2}\left(b_{2}+\theta\right)\right]}$.

\section{Figures}

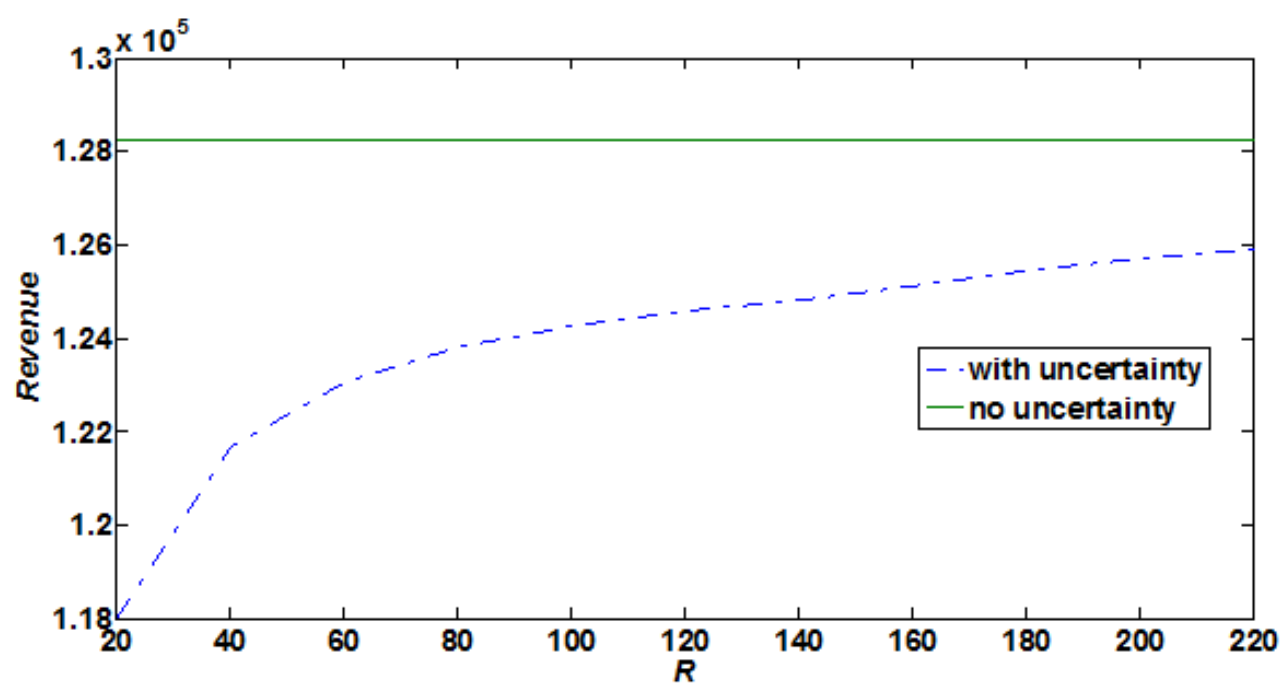

Fig.A1 The retailer's revenue as a function of $\boldsymbol{R}$ 


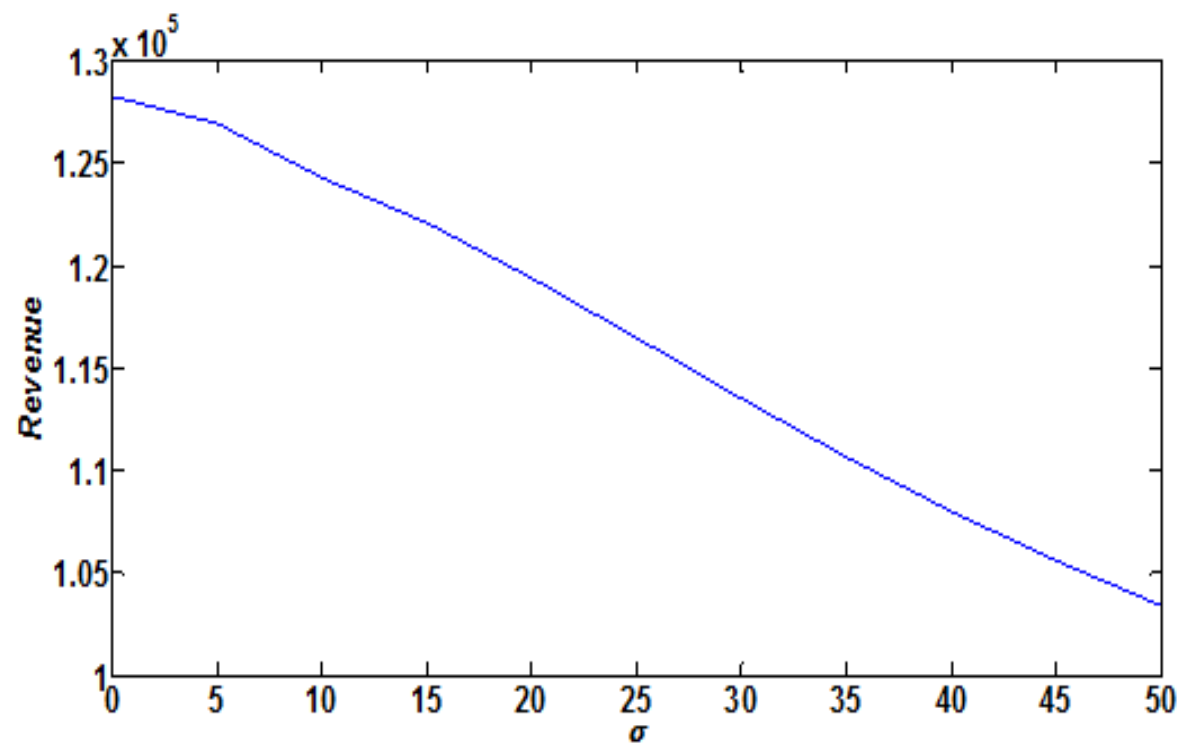

Fig.A2 The retailer's revenue as a function of the standard deviation $\sigma$

Figs.A1 and A2 show that the expected revenue with an uncertainty online demand is lower than the revenue with a deterministic online demand. And the expected revenue increases with the retailer's risk tolerance but decreases with the standard deviation.

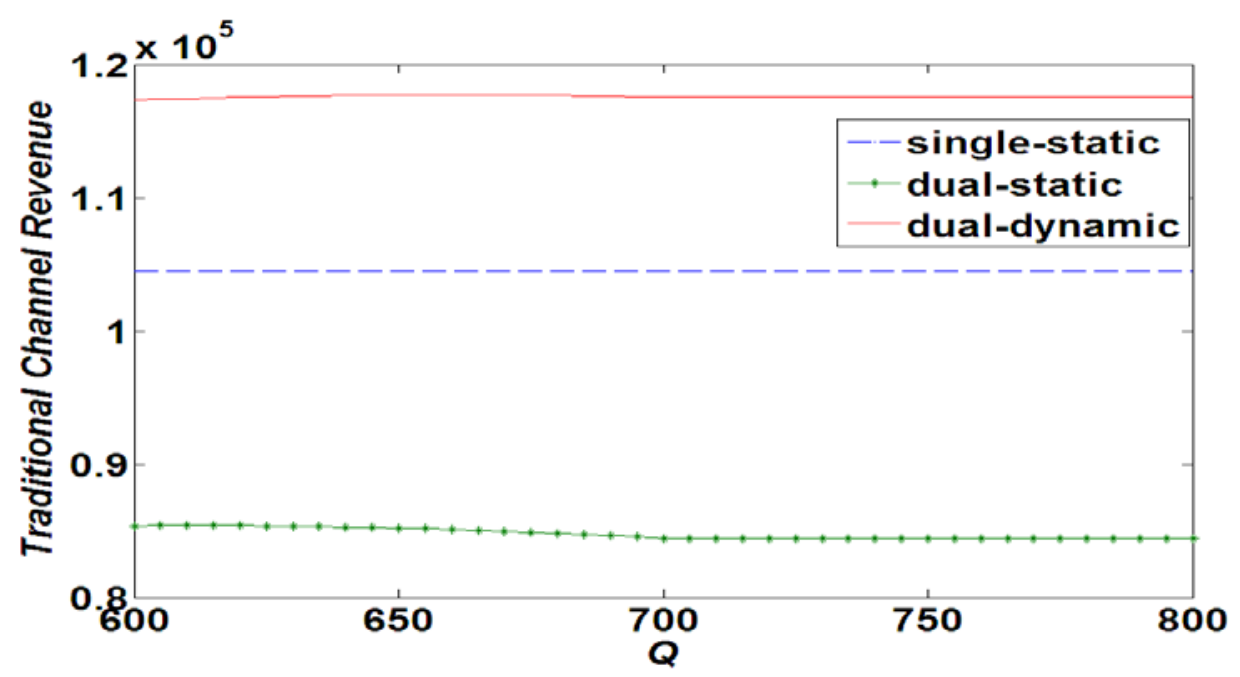

Fig.A3Revenue of the traditional channelas a function of $Q$ 


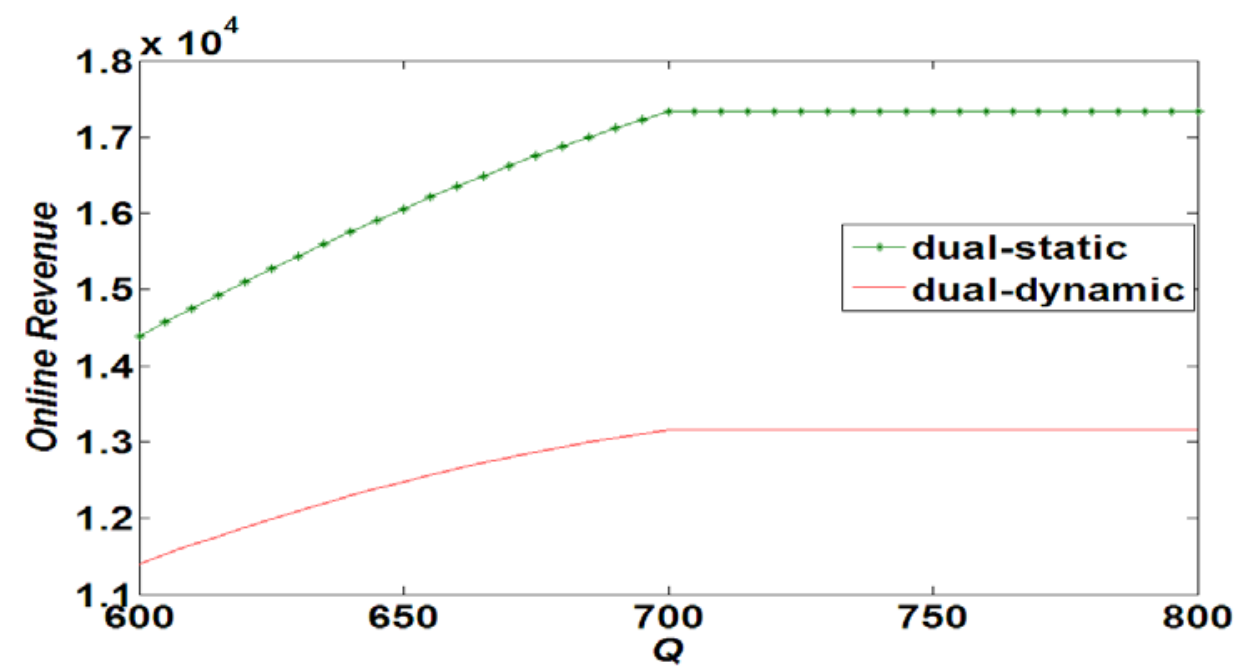

Fig.A4Revenue of the online channel as a function of $Q$

Figs.A3 and A4 illustrate the change of the revenues of the traditional and the online channels, respectively. When the retailer uses static price for the traditional channel, the revenue of the traditional channel decreases with the introduction of the online channel. The resultsuggests the existence of channel conflict. But when dynamic pricing is used for the traditional channel, the revenue of the traditional channel and the total revenue increasebut the revenue of the online channel decreases.In other words, dynamic pricing mitigates channel conflict. 